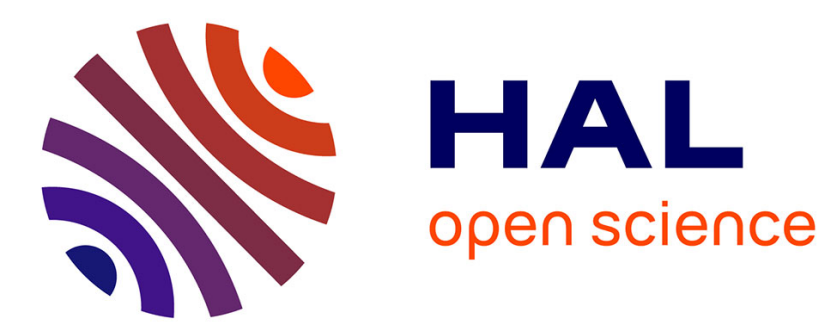

\title{
Mise au point - L'ionisation de contact
}

J.B. Moreau

\section{- To cite this version:}

J.B. Moreau. Mise au point - L'ionisation de contact. Revue de Physique Appliquée, 1968, 3 (3), pp.286-304. 10.1051/rphysap:0196800303028600 . jpa-00242863

\section{HAL Id: jpa-00242863 https://hal.science/jpa-00242863}

Submitted on 1 Jan 1968

HAL is a multi-disciplinary open access archive for the deposit and dissemination of scientific research documents, whether they are published or not. The documents may come from teaching and research institutions in France or abroad, or from public or private research centers.
L'archive ouverte pluridisciplinaire HAL, est destinée au dépôt et à la diffusion de documents scientifiques de niveau recherche, publiés ou non, émanant des établissements d'enseignement et de recherche français ou étrangers, des laboratoires publics ou privés. 


\title{
MISE AU POINT
}

\section{L'IONISATION DE GONTAGT}

\author{
Par J. B. MOREAU, \\ Laboratoire de Radioélectricité Générale du Conservatoire National des Arts et Métiers $\left.{ }^{\mathbf{1}}\right)$. \\ (Reçu le $1^{\mathrm{er}}$ juillet 1968.)
}

\begin{abstract}
Résumé. - L'ionisation de contact consiste à ioniser un atome (alcalin en général) au contact d'une surface chaude. Ce phénomène associé à l'émission thermo-électronique conduit à la réalisation d'émetteurs mixtes d'électrons et d'ions. Deux théories tentent d'expliquer ce phénomène : l'une (Rasor) suppose que les particules adsorbées sur la surface existent dans deux états distincts, atomique ou ionique; la seconde (Gyftopoulos) considère qu'il n'existe qu'un type de particules adsorbées (les adatomes) dont les liaisons avec les atomes du substrat sont partiellement covalentes et partiellement électrovalentes. Nous présentons en parallèle ces deux théories pour en dégager les aspects essentiels. De même que la loi de RichardsonDushman, la loi de Saha-Langmuir qui exprime quantitativement l'émission ionique, est une fonction du potentiel de sortie $\varphi$ du matériau émetteur. Son exploitation serait simple si $\varphi$ était constant. En fait, $\varphi$ dépend du taux de recouvrement $\theta$ de la surface par le métal alcalin et les principales difficultés consistent à exprimer $\varphi$ en fonction de $\theta$ et $\theta$ en fonction des paramètres accessibles à l'expérience. Les résultats expérimentaux confirment les expressions théoriques de Rasor ou Gyftopoulos, mais ne sont pas assez précis pour apprécier le degré de validité de l'une ou l'autre théorie.
\end{abstract}

Abstract. - Contact ionization occurs when an atom (usually alkaline) is ionized at the contact of a hot surface. This phenomenon combined with the thermo-electronic emission leads to the realization of mixed emitters of electrons and ions. Two theories attempt to explain this phenomenon, - one (Rasor) assuming that the particles adsorbed on the surface exist in an either atomic or ionic state, - the other (Gyftopoulos) maintaining that there is only one type of adsorbed particles (the adatoms), held onto the surface by bonds that are partially covalent, partially electrovalent. We draw a parallel between these two theories and try to bring forward their most essential aspects. Just as Richardson-Dushman's law, Saha-Langmuir's law, which expresses quantitatively the emission of ions, is a function of the work function $\varphi$ of the emitting material. This law could readily be used if $\varphi$ was constant. However, $\varphi$ is dependent on the degree of coverage $\theta$ of the surface by the alkaline metal, and the main difficulties consist in defining $\varphi$ as a function of $\theta$, and $\theta$ as a function of the parameters accessible to the experiment. The experimental results confirm Rasor's and Gyftopoulos' theoretical expressions ; they are, however, too vague to allow an appreciation of their value.

Pendant près de cinquante ans, la radioélectricité a pu se développer grâce à la mise en application de l'effet thermoélectronique. Mais, pour concevoir des tubes radioélectriques exploitant cet effet, il faut, bon gré mal gré, obéir à la nature, et se contenter de particules de charge négative. Il est pourtant possible de concevoir des systèmes radioélectriques utilisant

(1) Ce laboratoire travaille en contact étroit avec le laboratoire de recherche de la Compagnie Française Thomson Houston-Hotchkiss Brandt. des particules de charge positive, voire même simultanément des particules de charge opposée. Si l'on pouvait disposer d'un émetteur de charges positives aussi simple que le filament chauffé émettant des électrons, on pourrait tenter de réaliser des tubes dont le fonctionnement, tel que le laisse prévoir la théorie, serait basé sur l'interaction de deux faisceaux de charge opposée, voire même des tubes comportant un plasma résultant du mélange intime et de la thermalisation de ces faisceaux... La propulsion électrique dont l'intérêt s'est manifesté dès le début des études spa- 
tiales bénéficierait également d'une telle source d'ions qui résoudrait vraisemblablement le difficile problème de la neutralisation de la charge d'espace. Bien sûr, l'obtention de sources thermiques de particules positives n'est qu'une condition nécessaire; une réalisation technique exige la synthèse harmonieuse d'un grand nombre d'applications de phénomènes physiques et non pas d'un seul... Mais, si le principal fait défaut, la faillite est certaine.

L'ionisation par contact est le phénomène physique qui semble le plus utilisable pour créer ces particules positives. Nous avons pris, comme point de départ de cette étude, trois travaux de base comportant une abondante bibliographie, l'article de Zandberg et Ionov (1959), celui de Eberhagen (1960) et l'ouvrage de Kaminski (1965). Nous y renvoyons le lecteur pour toutes les références antérieures à 1960, sauf pour les articles de Langmuir, qui reste le fondateur de cette théorie. Depuis 1960, de nombreux articles sont apparus et nous tentons d'en présenter une liste aussi complète que possible. Nous avons extrait de ces articles les figures et tableaux les plus caractéristiques, de façon à aider le lecteur intéressé non pas seulement par la compréhension du phénomène, mais aussi et surtout par ses applications. Ce lecteur a besoin d'exemples nombreux lui permettant de juger si les performances de tels ou tels matériaux permettent d'espérer l'application qu'il a en vue. Les travaux, dont nous rendons compte, ont été effectués en grande partie pour étudier la conversion thermo-ionique (Rasor (1964), Houston (1962), Rittner (1962)) et la propulsion électrique (Gilleo et Kash (1961), Garvin et Wilson $(1965,1966))$; mais certaines références concernent des recherches fondamentales sur la physique des plasmas. Des machines destinées à fournir du plasma alcalin pour y étudier notamment la propagation des ondes ont été réalisées ces dernières années (Rynn (1960), Wada et Knechli (1961), E. Guilino (1963), Enriques et Magistrelli (1964), Doucet (1967), Moreau (1968), Felden et al. (1966)).

On peut aborder le problème par deux chemins fort différents. On peut imaginer que le métal est bordé par un plasma en équilibre thermodynamique, et décrire le phénomène d'échange de particules entre ces milieux grâce aux méthodes de la thermodynamique. Il est possible d'obtenir ensuite des renseignements sur les phénomènes hors d'équilibre, lorsque l'on trouve à côté du métal, non pas un plasma, mais un système d'électrodes exerçant des champs électriques. Une étude suivant cette approche a été faite par Carabateas (1962), Gadzuk (1965) et plus récemment par Maugis (1967). On peut, tout au contraire, progresser à partir du phénomène individuel, en tentant une théorie cinétique de l'émission; c'est ainsi que procèdent Rasor (1963-1964), Levine et Gyftopoulos (1964) et plusieurs autres. Cette méthode est beaucoup moins rigoureuse que la méthode thermodynamique. Mais elle a l'avantage de décrire et de tenter d'interpréter les phénomènes tels qu'ils se pas- sent effectivement, surtout dans les applications que nous avons en vue, où l'équilibre ne sera pratiquement jamais réalisé. Gette méthode a été perfectionnée en tenant compte des concepts de la mécanique quantique par Bennett et Falicon (1966) et par Kaplit et al. (1967), mais nous ne nous y arrêtons pas, les résultats expérimentaux étant trop imprécis pour justifier une telle étude.

1. Le phénomène individuel. - Un atome adsorbé sur la surface d'un métal peut être désorbé soit à l'état d'atome, soit à l'état d'ion positif. L'électron séparé de l'atome est absorbé par le métal. Un tel phénomène peut s'interpréter en première approximation par l'ionisation de l'atome adsorbé suivie de la désorption de l'ion. Admettons, en première approximation, que l'énergie de désorption d'un atome $E_{\mathrm{a} 0}$ et celle d'un ion $E_{\mathrm{i} 0}$ soient assez peu différentes $\left({ }^{2}\right)$; nous reviendrons dans la suite sur cette hypothèse dont la validité est sujette à caution. On peut alors calculer aisément le bilan énergétique du processus; on doit fournir à l'atome l'énergie d'ionisation $I$, mais on récupère l'énergie d'extraction d'un électron $e \varphi_{0}$ lorsque l'électron, libéré de l'atome, entre dans le métal; si $I$ est inférieur à $e \varphi_{0}$, le bilan sera bénéficiaire et le processus peut se produire spontanément sans intervention du milieu extérieur. On doit donc chercher des métaux à fort potentiel de sortie et des atomes à faible potentiel d'ionisation; des atomes alcalins comme le césium ou le potassium, adsorbés sur des métaux réfractaires tels le tungstène ou le tantale, constituent un bon exemple. On peut imaginer le phénomène inverse : un atome adsorbé prend un électron au métal, puis est désorbé sous forme d'ion négatif (Van Goeler et al. (1966)). Ce phénomène est peu intéressant pour les applications que nous envisageons; ces ions sont de même signe que les électrons, donc n'ont aucun intérêt pour compenser la charge d'espace d'un faisceau d'électrons; d'autre part, les atomes ainsi ionisables sont très électronégatifs (halogènes, oxygène) et, surtout à chaud, détruisent rapidement la surface métallique sur laquelle ils s'ionisent (Kaminski (1960)). Signalons, enfin, que des expériences d'ionisation de contact sur des isolants tels que l'alumine (Garvin et al. (1965)) ont été signalées; mais il est probable que le mécanisme est différent car l'isolant ne peut éliminer les électrons captés; les courbes expérimentales ont d'ailleurs une allure différente de celles obtenues avec des métaux.

La mise en évidence du phénomène est simple. Elle a été faite par Taylor et Langmuir (1933) à l'aide d'un tube de verre contenant deux filaments et un collecteur muni de deux anneaux de garde comme

${ }^{2}$ L Les indices o correspondent au fait que ces grandeurs sont des caractéristiques du phénomène individuel. Nous verrons que les choses se compliquent avec une surface recouverte de nombreux atomes. 


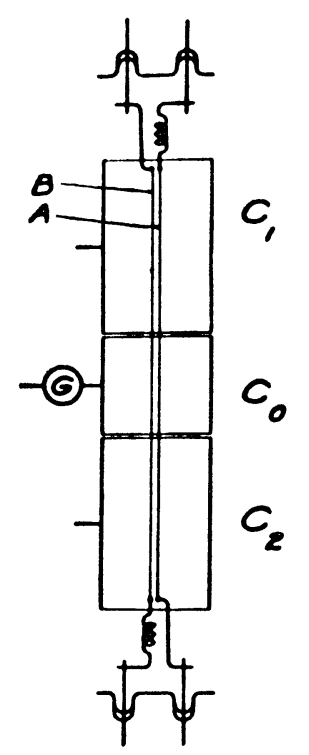

FIg. 1. - Tube de Taylor et Langmuir (1933).

le montre la figure 1. Les deux filaments sont destinés à faire des mesures du taux de recouvrement $\theta$ de l'un des filaments par des atomes adsorbés; nous verrons au $\S 4$ le principe de cette mesure. En chauffant seulement l'un des filaments et en le polarisant négativement par rapport au collecteur, on constate qu'un courant d'ions circule du filament vers la plaque. Ce courant dépend de la température du filament (supérieure à $900 \mathrm{oK}$ ) et de la pression de vapeur dans l'enceinte (de l'ordre de $10^{-6}$ à $10^{-5}$ torr dans les expériences de Langmuir). Taylor et Langmuir ont eu le mérite d'entreprendre une étude quantitative et c'est là une performance beaucoup plus difficile que la simple mise en évidence du phénomène; la figure 2 , extraite d'un article de Lieb et Kitrilakis (1966), montre que la méthode mise au point par Langmuir reste actuelle; seule, la technologie s'est améliorée. Elle est cependant limitée aux basses pressions de césium pour éviter l'amorçage d'arc et nous verrons au $\S 4$ d'autres méthodes de mesure destinées à pallier cet inconvénient.

En fait, le processus énergétique qui a été ébauché doit être perfectionné, comme l'a fait Rasor (1963, 1964). Le passage de l'état « atome adsorbé » à l'état « ion adsorbé » nécessite une énergie $E_{0}$ qui n'est pas accessible directement à l'expérience, car c'est un phénomène superficiel. Mais le principe de l'état initial et de l'état final conduit à un cycle ( fig. 3) qui permet d'exprimer ce paramètre essentiel à l'aide d'autres paramètres accessibles à l'expérience. Considérons un atome éloigné de la surface qui constitue l'état initial du système, et un ion et un électron également éloignés de la surface qui constitue l'état final. On peut passer de deux façons de cet état initial à cet état final :
- adsorption de l'atome $\left(-E_{\mathrm{a} 0}\right)$, ionisation sur la surface $\left(+E_{0}\right)$, désorption de l'ion $\left(+E_{\mathrm{i} 0}\right)$, émission de l'électron $\left(+e \Phi_{0}\right)$, ce qui correspond à la dépense d'énergie : $-E_{\mathrm{a} 0}+E_{0}+E_{\mathrm{i} 0}+\mathrm{e} \Phi_{0}$,

- ionisation de l'atome libre qui correspond à la dépense d'énergie $I$.

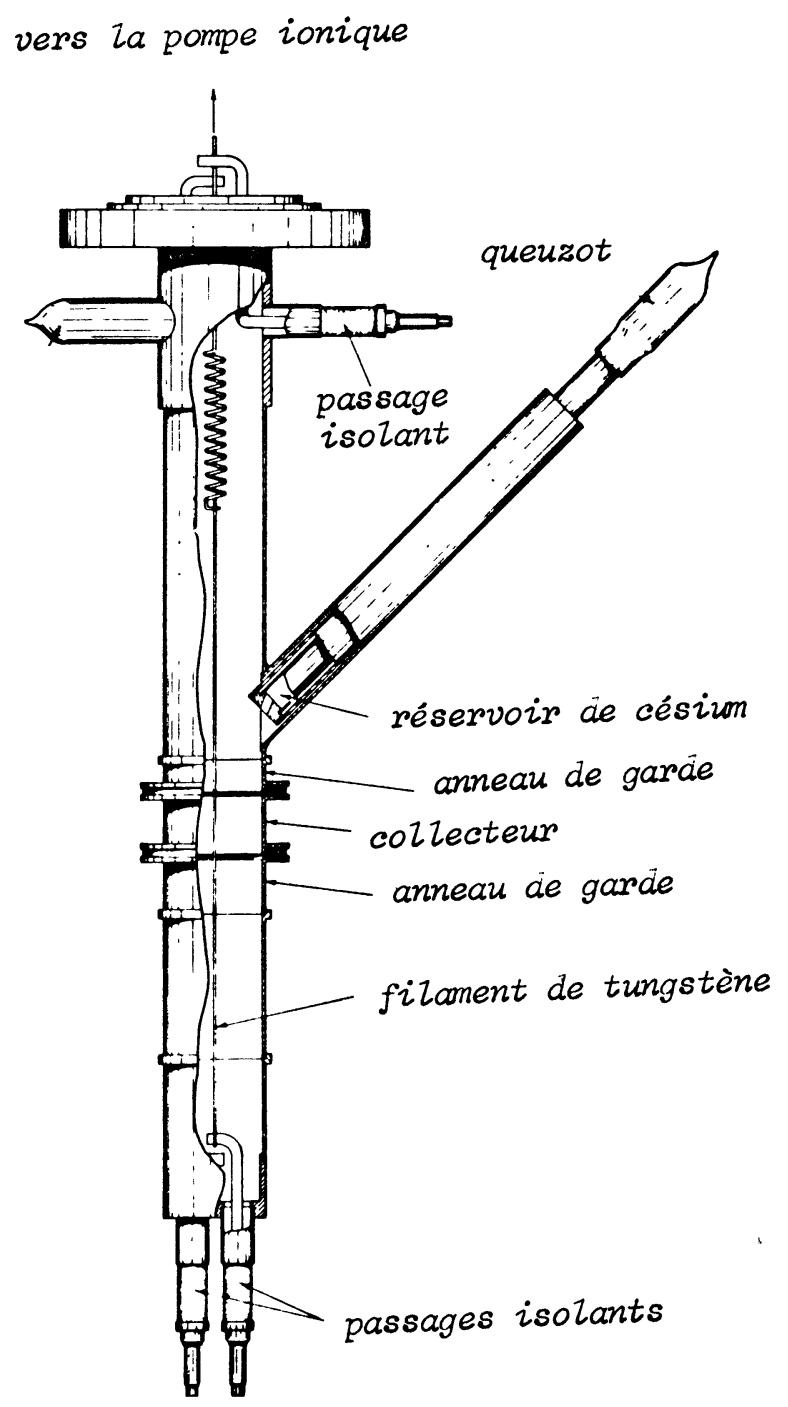

FIG. 2.

Tube de mesure de D. Lieb et S. S. Kitrilakis (1966).

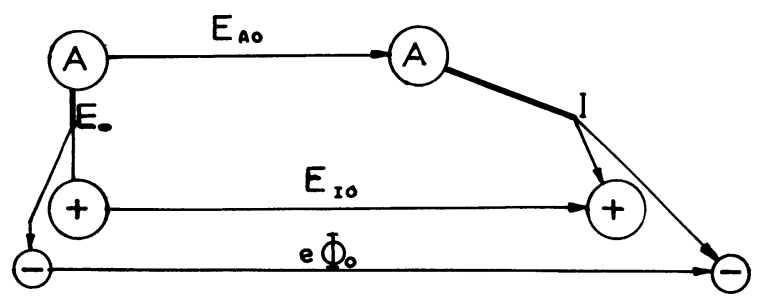

FIG. 3. - Cycle de Rasor. 
La dépense d'énergie étant la même dans les deux cas, on a :

$$
E_{0}=E_{\mathrm{a} 0}-E_{\mathrm{i} 0}+I-e \Phi_{0}
$$

ce qui permet d'exprimer $E_{0}$ inaccessible à l'expérience en fonction de grandeurs plus ou moins accessibles. On peut représenter l'état énergétique des particules adsorbées dans un diagramme tel que celui de la figure 4 qui correspond au cas de l'adsorption du
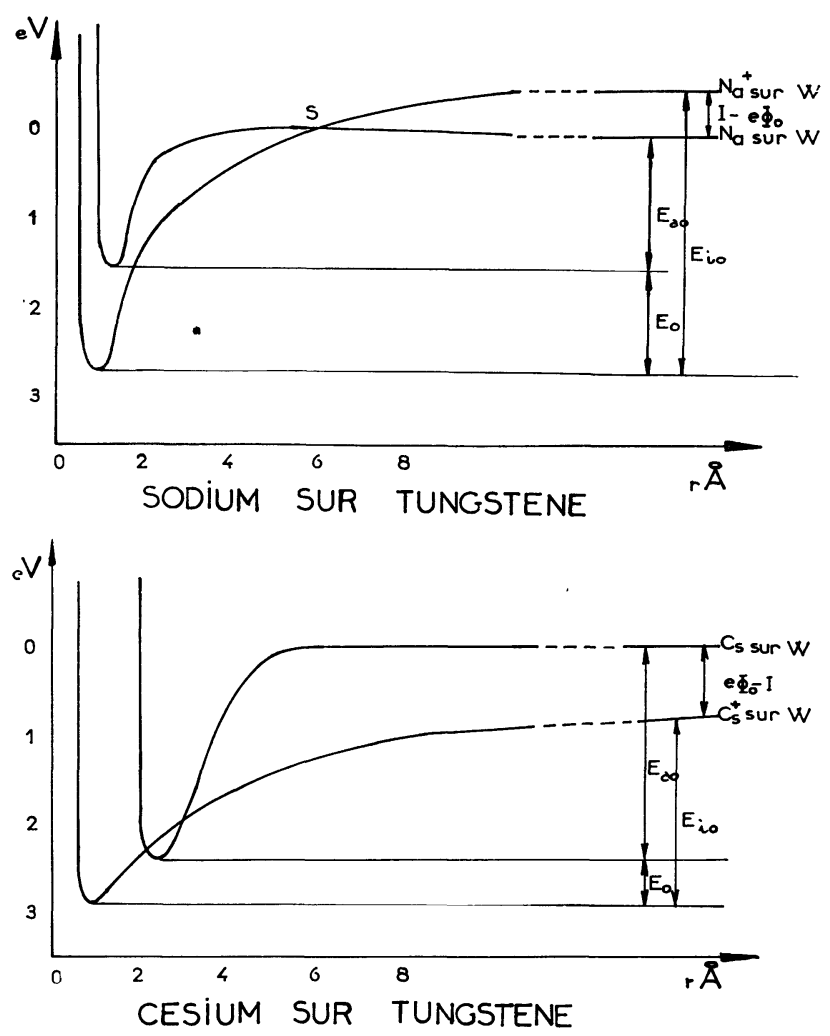

FIG. 4. - Diagramme d'énergie Rasor.

sodium et du césium sur le tungstène (Kaminski (1965)). On voit ainsi la signification des énergies utilisées dans le cycle de Rasor et on remarque de plus que le signe de $E_{0}$ n'est pas lié au signe de $e \Phi_{0}-I$. Si ce dernier est positif, l'atome sera désorbé préférentiellement sous forme ionique (césium). Au contraire, s'il est négatif, il y aura recombinaison de l'ion et de l'électron au passage du point $\mathrm{S}$ (l'atome choisit le niveau d'énergie le plus bas) et désorption sous forme atomique. Cela montre la complexité du phénomène et le danger de raisonnement simpliste tel que celui du début de ce paragraphe.

L'hypothèse précédente suppose l'existence, sur la surface du métal, de deux familles de particules, des ions et des atomes en interaction. Ce processus de raisonnement ne choque pas le physicien des plasmas, habitué à considérer une famille de neutres et une famille d'ions. Les atomes sont retenus par des liaisons de covalence, tandis que les ions sont retenus par des liaisons électrovalentes qui entraînent l'apparition d'un doublet électrique, donc d'une double couche sur la surface. C'est l'hypothèse fondamentale sur laquelle s'appuie Rasor (1964) qui suppose ainsi trois types de liaisons : ion-substrat, atome-substrat, auxquelles s'ajoutera, lorsque nous passerons des phénomènes individuels aux phénomènes collectifs, la liaison entre les atomes et ions adsorbés.

Mais on peut partir d'une hypothèse quelque peu différente, suivant laquelle un seul type de particule que nous baptisons " adatomes " est adsorbé; la désorption a lieu à l'état d'ion ou à l'état d'atome avec des probabilités qui sont conditionnées par la différence des énergies finales, qui apparaît sur la figure 5 . Seule, $e \Phi_{0}-I$ et $E_{\mathrm{a} 0}$, énergie de désorption à l'état atome, joue un rôle. Cette hypothèse est celle adoptée

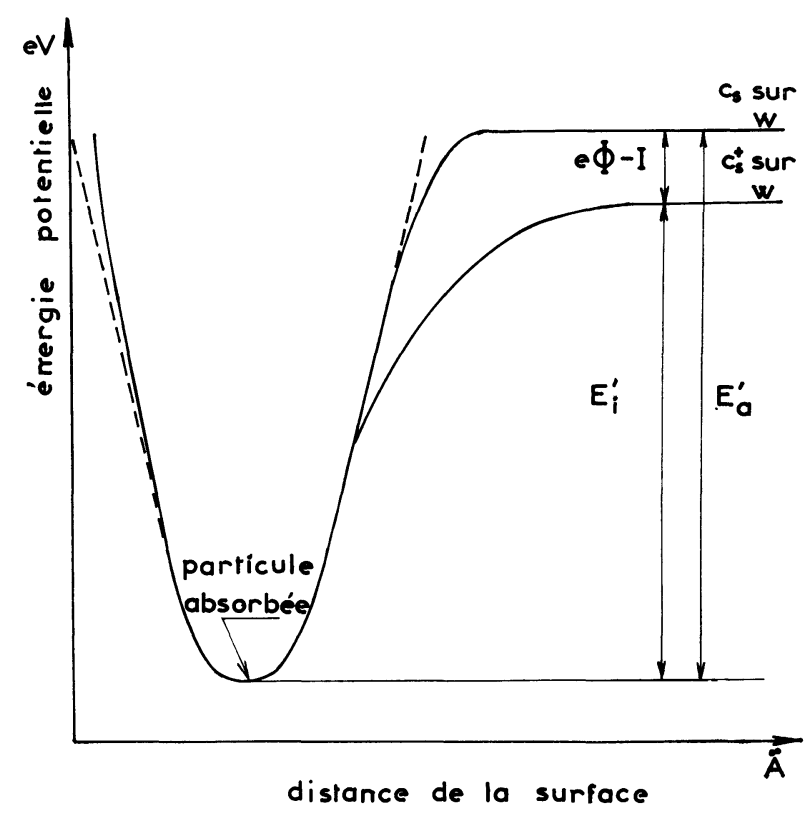

FIG. 5. - Diagramme d'énergie Levine.

par Levine et Gyftopoulos (1964); elle suppose l'existence de deux types de liaison : adatome-substrat et adatome-adatome. Mais, pour rendre compte des phénomènes, il est nécessaire de supposer que la liaison adatome-substrat est partiellement covalente et partiellement électrovalente (Gomer (1966)), entrâ̂nant ainsi l'existence d'un doublet électrique. Le calcul complet de l'échange de charge qui intervient dans une telle liaison est fort délicat (Gyftopoulos et Steiner (1967)) et fait appel à de nombreux paramètres malaisés à déterminer. Nous retrouverons ces deux méthodes de raisonnement plus tard.

Les différentes énergies qui entrent en jeu sont donc l'énergie d'ionisation $I$ de l'atome libre, les énergies de désorption $E_{\mathrm{a} 0}$ et $E_{\mathrm{i} 0}$ d'un atome ou d'un ion, et le travail de sortie $e \varphi_{0}$. Ce dernier paramètre est une grandeur caractéristique de la surfące, sa valeur dé- 
pend donc de l'état de la surface; nous calculerons celle-ci en présence d'atomes adsorbés au $\S 3$. Par contre, les valeurs des autres énergies sont connues ou peuvent se calculer si l'atome ou l'ion est isolé sur la surface; dans ce cas, l'énergie résulte de l'interaction avec le substrat; elle est bien définie. Nous avons groupé dans trois tableaux les valeurs des principales grandeurs physiques qui interviennent dans la suite.

Le tableau I donne les caractéristiques des métaux alcalins et du baryum. Il comporte le rayon ionique $r_{1}$, l'énergie d'ionisation $I$ et la chaleur de sublimation $h_{\mathrm{a}}$ qui servira au calcul de l'énergie de désorption de l'atome $E_{\mathrm{a} 0}$, laquelle dépend à la fois du corps adsorbé et du substrat. On a aussi indiqué $e \Phi_{\mathrm{A}}$, travail de sortie du métal alcalin pur. Cette valeur est importante pour la suite.

Le tableau II donne la chaleur de sublimation $h_{\mathrm{s}}$, le travail de sortie du métal pur $e \Phi_{0}$ et la température

\section{TABLEAU I}

Constantes Physiques Des mÉTAuX ADSORbÉS

\begin{tabular}{|c|c|c|c|c|c|}
\hline & $\begin{array}{c}\text { RAYON } \\
\text { IONIQUE } \\
r_{\mathrm{i}} \AA \\
\end{array}$ & $\begin{array}{c}\text { Chaleur } \\
\text { DE } \\
\text { SUbLimation } \\
h_{\mathrm{a}} \mathrm{eV} \\
-\end{array}$ & $\begin{array}{c}\text { Travail } \\
\text { DE SORTIE } \\
e \Phi_{\mathrm{A}} \mathrm{eV}\end{array}$ & $\begin{array}{c}\text { ÉNERGIE } \\
\text { D'IONISATIION } \\
I \text { eV } \\
-\end{array}$ & $\begin{array}{c}\text { MAILle } \\
\text { GRISTALLINE } \\
\AA \\
\AA\end{array}$ \\
\hline Lithium & 0,6 & 1,66 & 2,49 & 5,36 & 3,508 \\
\hline Sodium & 0,95 & 1,12 & 2,28 & 5,12 & 4,291 \\
\hline Potassium & 1,33 & 0,92 & 2,24 & 4,32 & 5,211 \\
\hline Rubidium .. & 1,48 & 0,84 & 2,09 & 4,16 & 5,631 \\
\hline Césium & 1,69 & 0,8 & 1,81 & 3,87 & 6,062 \\
\hline Baryum & 1,35 & 1,8 & 2,48 & 5,19 & 5,019 \\
\hline
\end{tabular}

TABLEAU II

Constantes physiques des métauX De TRANSition

\begin{tabular}{|c|c|c|c|c|}
\hline & $\begin{array}{c}\text { GHALEUR DE } \\
\text { SUBLIMATION } \\
h_{\mathrm{s}} \mathrm{eV} \\
\end{array}$ & $\begin{array}{c}\text { Travail } \\
\text { DE SORTIE } \\
e \Phi_{0} \mathrm{eV}\end{array}$ & $\begin{array}{c}\text { Température } \\
\text { DE FUSION } \\
{ }^{\circ} \mathrm{K} \\
-\end{array}$ & $\begin{array}{c}\text { MaILle } \\
\text { CRISTALLINE } \\
\AA \\
-\end{array}$ \\
\hline Tantale & 8,11 & 4,19 & 3270 & 3,303 \\
\hline Tungstène & 8,68 & 4,62 & 3653 & 3,165 \\
\hline Niobium & 7,71 & 4,01 & 2760 & 3,301 \\
\hline Molybdène ... & 6,84 & 4,38 & 2883 & 3,147 \\
\hline Platine ....... & 5,85 & 5,32 & 2042 & 3,924 \\
\hline Rhénium ..... & 8,07 & 5,1 & 3453 & $\begin{array}{l}2,765 \\
4,470\end{array}$ \\
\hline
\end{tabular}

TABLEAU III

ÉNERGIES DE DÉSORPTION $E_{\mathrm{a} 0}$ en eV

$\begin{array}{cccccc}\text { Lithium } & \text { Sodium } & \text { Potassium } & \text { Rubidium } & \text { CÉsium } & \text { Baryum } \\ - & - & - & - & - & - \\ 2,54 & 2,08 & 1,89 & 1,81 & 1,76 & 2,64 \\ 2,46 & 2,02 & 1,83 & 1,75 & 1,71 & 2,56 \\ 2,26 & 1,86 & 1,68 & 1,61 & 1,57 & 2,35 \\ 2,45 & 2,02 & 1,83 & 1,75 & 1,7 & 2,55 \\ 2,4 & 1,97 & 1,78 & 1,71 & 1,66 & 2,5 \\ 2,08 & 1,71 & 1,55 & 1,48 & 1,45 & 2,17\end{array}$


de fusion $T_{\mathrm{f}}$ de quelques métaux qui peuvent être utilisés soit comme émetteur, soit comme élément constitutif d'un propulseur ou d'une machine à plasma et ainsi être en contact avec les vapeurs ionisables. Il est clair que le métal ne sera techniquement utilisable que si $T_{\mathrm{f}}$ et $h_{\mathrm{s}}$ sont très élevés.

Le tableau III donne la valeur de l'énergie de désorption d'un atome $E_{\mathrm{a} 0}$ pour les métaux alcalins et le baryum associés aux principaux métaux réfractaires utilisés comme émetteur. Pauling (1960) a montré, en effet, que l'énergie de désorption $E_{\mathrm{a} 0}$ d'un atome était proportionnelle à la moyenne géométrique des chaleurs de sublimation du métal adsorbé et du métal substrat. Le facteur de proportionnalité est compris entre 0,5 et 1 (Levine (1963)). Pour le césium sur le tungstène, ce facteur est égal à 0,67 , chiffre que nous avons utilisé pour calculer les valeurs de $E_{\mathrm{a} 0}$ pour les autres couples de métaux. La valeur de $E_{\mathrm{a} 0}$ ainsi calculée pour le césium sur le tungstène est inférieure à celle mesurée par Taylor et Langmuir (1937), Kœnig et Pigford (1966) (de l'ordre de 2,79 eV) et par Swanson (1964) (de l'ordre de $3 \mathrm{eV}$ ).

Nous devrions, en outre, disposer des valeurs de $E_{\mathrm{i} 0}$ pour exploiter le cycle de Rasor; en première approximation, nous pouvons considérer que le travail des forces coulombiennes entre l'ion et son image est important, au point de négliger l'effet des forces de Van der Waals. En calculant $E_{\mathrm{i} 0}$ par cette méthode, on trouve :

$$
E_{\mathrm{i} 0}=e^{2} / 8 \pi \varepsilon_{0} r_{\mathrm{i}}
$$

ce qui conduit à des valeurs de $E_{\mathrm{i} 0}$ du même ordre que $E_{\mathrm{a} 0}$. Taylor (1937) et Kœnig (1966) obtiennent également une valeur de $E_{\mathrm{i} 0}$ voisine de $2,05 \mathrm{eV}$, donc légèrement inférieure à $E_{\mathrm{a} 0}$. Scheer et Fine (1962), et Perel et al. (1965) trouvent des valeurs beaucoup plus faibles, de l'ordre de 1,5 eV. Si la surface est propre, Scheer retrouve la valeur de Kœnig.

Indiquons en terminant que le cycle de Rasor reste évidemment valable si de nombreux atomes se trouvent sur la surface. Les divers paramètres sont modifiés, sauf $I$, mais le bilan est inchangé.

2. L'émission des ions et des électrons. - Considérons une pièce de métal à la température $T$ plongée dans un bain de vapeur alcaline. Nous appelons $\mu_{0}$ le flux d'atomes frappant l'unité d'aire de la surface. Si le gaz est une vapeur saturante en équilibre avec le métal alcalin en fusion à la température $T_{0}$, ce flux $\mu_{0}$ s'exprime, en supposant l'isotropie parfaite :

$$
\mu_{0}=n \sqrt{k T_{0} / 2 \pi M}
$$

$n$ est la densité de particules dans la vapeur et $M$ sa masse; si la pression $p$ est assez faible, on a :

$$
p=n k T_{\mathbf{0}}
$$

et par suite :

$$
\mu_{0}=p\left(T_{0}\right) / \sqrt{2 \pi M k T_{0}} .
$$

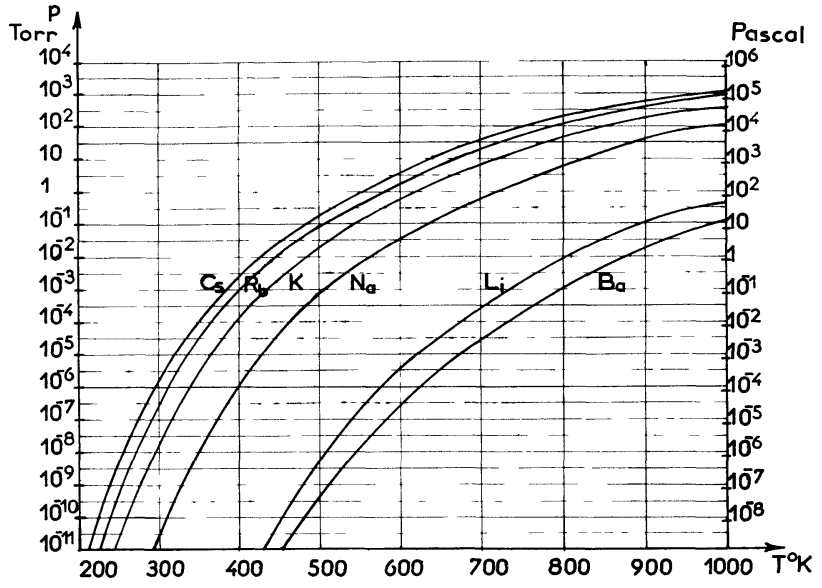

FIG. 6. - Pressions de vapeur.

Les courbes de la figure 6 donnent les valeurs des pressions de vapeur saturante des métaux alcalins et du baryum en fonction de la température $T_{\mathbf{0}}$ (Honig (1962)). Ainsi, avec une température $T_{0}=650 \mathrm{oK}$, le flux est de l'ordre de $4,25 \times 10^{25}$ atomes $/ \mathrm{m}^{2} / \mathrm{s}$ dans le cas du césium, ce qui correspond à une densité de courant de $6,8 \times 10^{6} \mathrm{~A} / \mathrm{m}^{2}$ si l'on suppose que chaque atome est désorbé à l'état ionisé.

On peut également envisager un bombardement de la surface du métal par un jet d'atomes issu d'un four terminé par un capillaire. Dans un tel cas, les méthodes classiques de la théorie cinétique (Boutry (1962)) permettront de déterminer $\mu_{0}$ en fonction de la température du four. Cette formule remplacera, dans la suite, la formule (2.3).

Examinons un atome qui est adsorbé sur la surface du métal; il se trouve dans le puits de potentiel de la figure 4. A un instant donné, nous trouvons $n_{\mathrm{a}}$ particules adsorbées par unité d'aire à l'état d'atome; nous allons faire l'hypothèse que cette collection de particules est en équilibre thermodynamique avec la surface à la température $T$; cela nous permet de déterminer le flux de particules désorbées, qui est égal à :

$$
\mu_{\mathrm{a}}=n_{\mathrm{a}} \nu_{\mathrm{a}} \exp -\frac{E_{\mathrm{a}}}{k T}
$$

$\nu_{\mathrm{a}}$ est la fréquence des oscillations dans le fond du puits de potentiel. Les $n_{\mathrm{a}}$ particules par unité d'aire se présentent $\nu_{\mathrm{a}}$ fois par seconde pour se désorber; l'exponentielle exprime la probabilité de franchissement de la barrière de potentiel $E_{\mathrm{a}}$. Au même instant, nous trouvons $n_{\mathrm{i}}$ particules adsorbées à l'état d'ions, ce qui nous conduit au flux d'ions désorbés :

$$
\mu_{\mathrm{i}}=n_{\mathrm{i}} \nu_{\mathrm{i}} \exp -\frac{E_{\mathrm{i}}}{k T} \text {. }
$$

Il nous faut chercher un ordre de grandeur des fréquences d'oscillations; on peut supposer qu'il s'agit de corpuscules de masse $M$ oscillant dans un puits de potentiel parabolique dont la figure 4 donne l'allure. 
Comme les ordres de grandeur des paramètres de l'ion et de l'atome sont les mêmes, on a $\nu_{a} \simeq \nu_{i}$ : $10^{12}$ hertz représente un ordre de grandeur satisfaisant, que confirment les études expérimentales de Scheer et Fine (1962), et de Kœnig et Pigford (1966).

Nous devons maintenant introduire les valeurs de $E_{\mathrm{i}}$ et de $E_{\mathrm{a}}$ (en suivant ici la théorie de Rasor). Nous connaissons ces valeurs si les atomes ou ions absorbés sont rares, donc s'ils interagissent peu; le $\S 1$ nous renseigne alors, tout au moins en ce qui concerne $E_{\mathrm{a}}$. Mais si les adatomes sont nombreux, ils vont interagir les uns avec les autres, et l'énergie nécessaire pour la désorption va être modifiée (Gyftopoulos et Steiner (1967)). Pour poursuivre cette étude, nous devons donner une définition du taux de recouvrement $\theta$. Considérons un atome alcalin, et plus précisément un atome de césium adsorbé sur la surface du tungstène. Il se place dans une position géométrique déterminée, définie par le réseau cristallin. Le césium et le tungstène cristallisent tous deux dans le système cubique centré, la maille du tungstène étant pratiquement deux fois plus petite que celle du césium (fig. 7).

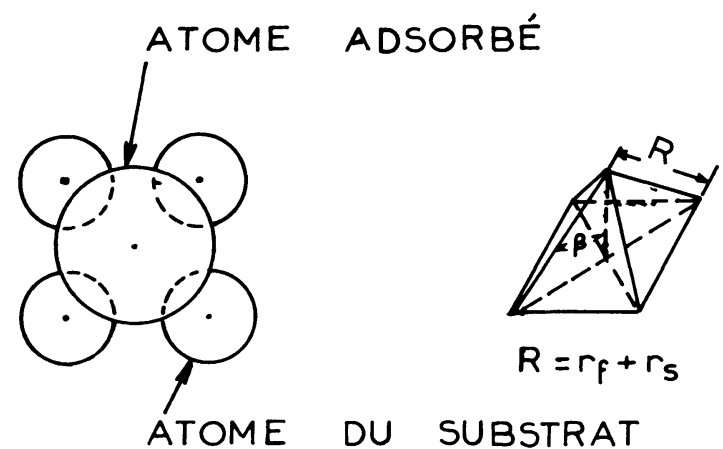

FIG. 7. - Position de l'atome adsorbé.

Les autres métaux alcalins et le baryum, ainsi que les principaux métaux réfractaires utilisés pour l'ionisation, cristallisent dans le même système cubique centré. Les dimensions de la maille cristalline sont indiquées sur les tableaux I et II. On peut vérifier que la maille du césium est pratiquement toujours deux fois supérieure à celle des métaux émetteurs. Il est clair que nous trouvons, par unité d'aire du métal, $N$ positions possibles; cela nous conduit à la notion de taux de recouvrement, $\theta$, défini comme le quotient du nombre d'atomes réellement adsorbés $n$ par le nombre de places $N$. Cette définition suppose que les atomes adsorbés forment d'abord une couche monoatomique complète avant d'en commencer une seconde. Cette hypothèse a été vérifiée par Mayer (1965) pour les métaux alcalins adsorbés sur un métal réfractaire. Il y a, bien sûr, deux cas à considérer :

$\theta=\frac{n}{N}<1$ : le recouvrement est incomplet, $\theta$ est inférieur à l'unité. Cela intervient aux tempéra- tures élevées. Le métal est recouvert d'une couche monoatomique incomplète,

$\theta>1$ : le recouvrement est complet, $\theta$ est supérieur à l'unité et nous sommes en présence d'une surface alcaline.

En fait, la détermination de $N$ est très délicate, car la surface métallique n'est évidemment pas plane, à l'échelle atomique. On peut néanmoins obtenir un ordre de grandeur de $N$. En la supposant plane, on trouve environ $3,5 \times 10^{14}$ atomes par $\mathrm{cm}^{2}$ pour le césium sur le tungstène, Taylor et Langmuir (1933) ont trouvé expérimentalement $4,8 \times 10^{14}$ atomes par $\mathrm{cm}^{2}$, ce qui traduit l'effet de la non-planéité.

Si $\theta$ est faible, les atomes adsorbés sont très éloignés et les formules $(2.4)$ et (2.5) sont valables avec les paramètres dont les valeurs figurent au $\S 1$. Mais au fur et à mesure que $\theta$ augmente, les atomes adsorbés se rapprochent et interagissent. Ge phénomène se traduit par l'introduction d'un coefficient $X$ dans les formules (2.4) et (2.5). $X$ est égal à l'unité pour $\theta$ faible, puis croît avec $\theta$. On conçoit la difficulté d'évaluer cette grandeur.

Un raisonnement basé sur la mécanique statistique a été fait par Warner (1961) et par Levine (1964). Il permet d'exprimer $X$ en fonction de $\theta$ par la relation :

$$
X=\frac{S}{S_{\mathrm{f}}} \exp \left[\theta \frac{\partial}{\partial \theta}\left(\log \frac{S}{S_{\mathrm{f}}}\right)\right] .
$$

Dans cette expression, $S_{\text {f }}$ représente la fraction de surface totale $S$ dont dispose une particule pour se déplacer librement. Warner utilise un modèle de particule du type boule de billard et obtient l'expression suivante :

$$
S_{\mathrm{f}}=S\left(1-a_{1} \theta\right)
$$

Pour le césium sur le tungstène, $a_{1}=1$ est une valeur raisonnable qui conduit cependant à surestimer la valeur de $S_{\mathrm{f}}$.

Si l'on utilise le modèle de la « cage » défini par Lennard-Jones (1937) pour les gaz très denses, la particule n'est libre de se mouvoir que sur la surface limitée par les particules les plus proches. L'expression de $S_{\mathrm{f}}$ devient :

$$
S_{\mathrm{f}}=S a_{\mathbf{2}}\left(1-\theta^{1 / 2}\right)^{2} .
$$

La théorie montre que $a_{2}=\pi$. Avec cette valeur, seules les valeurs de $\theta$ supérieures à $\left(1-a_{\curvearrowright}^{-1 / 2}\right)^{2}$ sont possibles pour que $S_{\mathrm{f}}<S$.

Si l'on pose $a_{2}=1$, la valeur de $S_{\mathrm{f}}$ déduite de la formule (2.8) sera trop faible. Assez arbitrairement, Levine fait ce choix et propose de prendre pour $S_{f}$ l'expression suivante qui est la moyenne géométrique des expressions (2.7) et (2.8) en faisant $a_{1}=a_{2}=1$ :

$$
S_{\mathrm{f}}=S(1-\theta)^{1 / 2}\left(1-\theta^{1 / 2}\right) \text {. }
$$


Nous donnons ci-dessous les valeurs de $X$ obtenues en adoptant cette dernière hypothèse :

$\begin{array}{cccccccccc}\theta & 0 & 0,1 & 0,2 & 0,3 & 0,4 & 0,5 & 0,6 & 0,7 & 0,8 \\ - & - & - & - & - & - & - & - & - & - \\ X & 1 & 2,8 & 3,43 & 5,8 & 11,5 & 27,3 & 84 & 435 & 11500\end{array}$

On voit que les hypothèses conduisant à ces valeurs de $X$ sont assez fragiles. Ce qu'il faut retenir, c'est que $X=1$ pour $\theta=0$ et tend vers l'infini si $\theta \rightarrow 1$. Ce paramètre $X$ sera indispensable au $\S 3$. Dans la suite de ce paragraphe, il n'est pas utile, les formules (2.4) et (2.5) n'intervenant que par leur rapport.

Nous devons enfin écrire qu'entre la famille des atomes adsorbés et celle des ions adsorbés il existe des échanges qui sont régis par une loi maxwellienne :

$$
\frac{n_{\mathrm{i}}}{n_{\mathrm{a}}}=g \exp -\frac{E}{k T}
$$

$g$ est le rapport des poids statistiques de l'ion et de l'atome; il est égal à $1 / 2$ dans le cas usuel des atomes alcalins qui ne possèdent qu'un seul électron sur la couche superficielle et dont l'ion a une structure de gaz rare.

Le fonctionnement permanent du montage exige que le flux total désorbé soit égal à $\mu_{0}$. Cela conduit à :

$$
\mu_{0}=\mu_{\mathrm{i}}+\mu_{\mathrm{a}} \text {. }
$$

En injectant les équations $(2.4),(2.5)$ et $(2.10)$ dans cette équation, on obtient :

$$
\mu_{\mathrm{i}}\left(1+\frac{1}{g} \exp \frac{E+E_{\mathrm{i}}-E_{\mathrm{a}}}{k T}\right)=\mu_{0}
$$

et en tenant compte de l'équation (1.1) qui traduit le cycle de Rasor :

$$
\mu_{\mathrm{i}}=\frac{\mu_{0}}{1+\frac{1}{g} \exp \left(\frac{I-e \Phi}{k T}\right)}
$$

Cette formule essentielle qui exprime la loi de Saha-Langmuir traduit les phénomènes de l'émission ionique. Le courant d'ion émis par une surface d'aire $S$ portée à la température $T$ et exposée à un flux d'atomes $\mu_{0}$ est donné par :

$$
I^{+}=S e \mu_{0} \frac{1}{1+\frac{1}{g} \exp \left(\frac{I-e \Phi}{k T}\right)}
$$

formule qu'il convient de rapprocher de celle de Richardson :

$$
I^{-}=S A T^{2} \exp (-e \Phi / k T)
$$

qui donne le courant électronique.

Ces deux formules doivent être exploitées simultanément; les paramètres $g, I, S$ et $T$ se déterminent facilement encore qu'il soit souvent difficile d'obtenir une surface à une température uniforme et de déter- miner avec précision l'aire de la surface émissive $\mathrm{S}$. $A$ est un paramètre fondamental de l'émission thermoélectronique qui vaut environ $1,2 \times 10^{6} \mathrm{~A} / \mathrm{m}^{2}$, si la surface est propre... Cette dernière remarque nous conduit au cœur même de la difficulté de cette étude; en effet, la surface est partiellement recouverte d'atomes adsorbés. Ce recouvrement qu'il nous faut maintenant examiner modifie probablement $A$, mais également $\Phi$, paramètre essentiel puisqu'il intervient dans les termes exponentiels.

3. Le potentiel de sortie du métal. - Il est clair que le potentiel de sortie dépend du taux de recouvrement de la surface $\theta$. Si $\theta$ est faible, nous sommes en présence de métal propre et la valeur de $\Phi$ est égale à $\Phi_{0}$, donnée dans les tables du premier paragraphe. Au contraire, si $\theta$ est égal à l'unité, le recouvrement est total, et l'on se trouve en présence d'une surface émissive constituée de métal alcalin, $\Phi$ est alors égal au potentiel de sortie $\Phi_{\mathrm{A}}$ de ce métal (cf. $\S 1$ ). Ainsi, $\Phi$ varie de $\Phi_{0}$ jusqu'à $\Phi_{\mathrm{A}}$ lorsque $\theta$ varie de 0 à 1 et nous devons tenter d'expliquer théoriquement ce phénomène.

Mais le paramètre $\theta$ est fort malaisé à employer. En fait, $\theta$ dépend de $T$, température du métal, et de $\mu_{0}$, flux d'atomes alcalins. Lorsque le métal est à basse température, il est recouvert de métal alcalin, $\theta$ est égal à l'unité; au contraire, lorsque $T$ est très élevé, la surface se nettoie, et $\theta$ tend vers 0 et ceci d'autant plus rapidement que $\mu_{0}$ est plus faible. Langmuir, à partir de résultats expérimentaux, a proposé une formule empirique qui conduit au réseau de courbes de la figure 8 donnant $\theta$ en fonction de $T$.

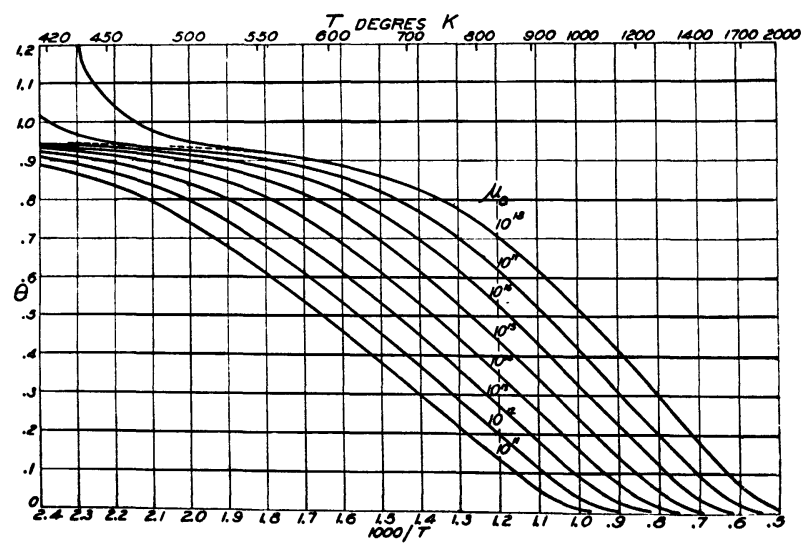

FIG. 8. - Variation de $\theta=f(T)$ (T. et L., 1933).

Commençons par chercher une interprétation de la variation de $\theta$ avec $T$. Pour cela, revenons à la définition de $\theta$ comme le fait Rasor (1964) et remplaçons les paramètres par leur valeur. Il vient :

$$
\theta=\frac{n_{\mathrm{i}}+n_{\mathrm{a}}}{N}=\frac{n_{\mathrm{i}}[1+2 \exp (E / k T)]}{N}
$$


et enfin :

$$
\theta=\frac{\mu_{0}}{N \vee X(\theta)} \frac{1+2 \mathrm{e}^{E / k T}}{\mathrm{e}^{-E_{\mathrm{j}} / k T}\left(1+2 \exp -\frac{e \Phi-I}{k T}\right)}
$$

$X(\theta)$ étant la fonction définie au $\S 2$, où figurent ses valeurs. La mise en œuvre du cycle de Rasor (1.1) et la connaissance de la relation entre $\varphi$ et $\theta$ qui sera étudiée dans la seconde partie de ce paragraphe permettent de calculer $\varphi$ à partir de $T$. Il est aisé de voir que, si $T$ est très faible, le produit $X(\theta) . \theta$ donné par l'équation (3.2) est très élevé; cela n'est possible, d'après le $\S 2$, que si $\theta$ est voisin de 1 . D'autre part, si $T$ est très élevé, la valeur de $\theta$ que donne l'équation (3.2) est faible; elle vaut :

$$
\theta_{\lim } \simeq \mu_{0} / N . v
$$

ce qui exprime que les atomes qui viennent frapper l'unité d'aire de la surface chaque seconde effectuent un aller-retour dans le puits de potentiel; c'est effectivement la durée minimum de contact. Enfin, on constate que, toutes choses égales par ailleurs, $\theta$ varie dans le même sens que $\mu_{0}$.

L'établissement de la relation de $\Phi$ avec $\theta$ constitue la partie la plus délicate de la théorie; de nombreux auteurs s'y sont exercés, notamment E. P. Gyftopoulos et al. $(1962,1964,1967)$ et $\mathrm{N}$. Rasor et al. $(1961,1963$, 1964). Les exposés théoriques reposent sur des idées intéressantes, mais il faut constater, trop souvent, la présence de nombreux paramètres ad hoc dont la valeur numérique est indéterminable; on peut certes l'ajuster expérimentalement, mais cela est peu convaincant dès qu'il y a plusieurs paramètres pour une courbe.

Le potentiel de sortie $\Phi(\theta)$ est évidemment égal à $\Phi_{0}$, potentiel de sortie du métal, si $\theta=0$ (métal propre); pour $\theta=1$, on se trouve en présence d'une couche continue de métal alcalin adsorbé; on fait alors l'hypothèse légitime que $\Phi(1)$ est égal au potentiel de sortie d'une couche massive de métal alcalin, soit $\Phi_{\mathrm{A}}$. Enfin, on suppose que l'effet de quelques atomes adsorbés ou de quelques trous dans la couche adsorbée complète est négligeable, ce qui signifie que $\mathrm{d} \Phi / \mathrm{d} \theta$ est nul, aux deux extrémités de l'intervalle. Reste à déterminer la fonction de $\theta$. Dans un premier article (1962), Gyftopoulos et Levine se bornent à décrire les faits par un polynôme, en posant :

$$
\Phi(\theta)=\Phi_{0}-\left(\Phi_{0}-\Phi_{\mathrm{A}}\right)\left(3 \theta^{2}-2 \theta^{3}\right) .
$$

Dans un travail ultérieur, il s'appuie sur des résultats de R. S. Mulliken (1955) pour décrire $\Phi(\theta)$ par une fonction de Morse (Slater (1963)) :

$$
\begin{array}{r}
\Phi(\theta)=\Phi_{0}-\left(\Phi_{0}-\Phi_{\mathrm{A}}\right)\left[2 \exp k\left(1-\frac{1}{\sqrt{ } \bar{\theta}}\right)\right. \\
\left.-\exp 2 k\left(1-\frac{1}{\sqrt{ } \bar{\theta}}\right)\right]
\end{array}
$$

les bases théoriques sont beaucoup plus sérieuses, mais le paramètre $k$ est inconnu et, une fois qu'il est déterminé expérimentalement, on constate que la différence entre les deux expressions précédentes est généralement inférieure aux erreurs expérimentales $(k=3$ constitue la valeur optimale).

Les expressions précédentes traduisent grosso modo le fait que la surface est partiellement une surface propre $\left(\Phi_{0}\right)$, partiellement une couche d'atomes adsorbés qui, liés les uns aux autres par des liaisons de covalence, constituent des zones où le potentiel est $\Phi_{A}$. Rasor et Warner (1964) reprenant les idées de Langmuir négligent ce premier effet et supposent que $\Phi$ reste égal à $\Phi_{0}$ tant que $\theta$ reste faible, seul domaine où ils souhaitent développer leur théorie.

Mais un autre phénomène se superpose au précédent; lorsqu'un atome de césium vient s'adsorber sur la surface, il donne naissance à un doublet électrique. En effet, l'électron de l'atome de césium a tendance à le quitter pour passer dans le métal réfractaire, plus électronégatif que lui (fig. 9). Ce dipôle, dont le moment électrique est de l'ordre du produit de la charge de l'électron par le rayon de l'atome (soit $M_{0} \simeq 10^{+29} \mathrm{~Gb} / \mathrm{m}$ ), crée un champ électrique qui favorise la sortie des électrons là où le métal est encore propre (fig. 9). Le potentiel du métal propre

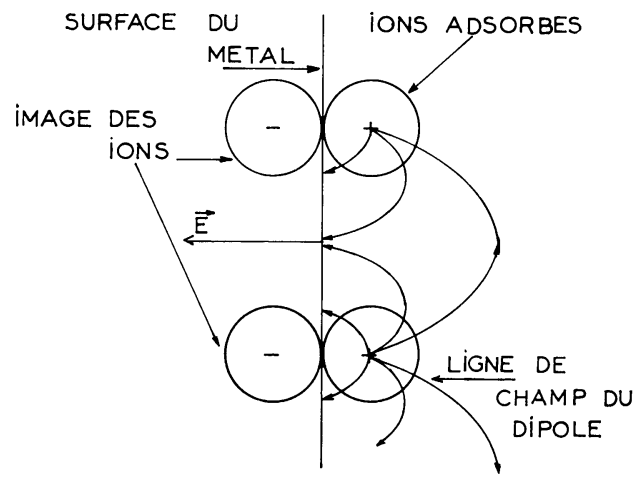

FIG. 9. - Champ des dipôles.

est donc diminué d'une certaine quantité, qui est proportionnelle, à la fois, au moment du dipôle élémentaire $M_{0}$, au nombre de dipôle par unité d'aire (c'est-à-dire à̀ $N \theta$ avec nos notations) et, enfin, à la superficie de surface propre encore disponible, c'est-àdire à $\left(\Phi-\Phi_{\mathrm{A}}\right) /\left(\Phi_{0}-\Phi_{\mathrm{A}}\right)$. Un calcul simple d'électrostatique conduit, pour ce terme correcteur, à :

$$
\Delta \Phi=-k \frac{N \theta}{2 \varepsilon_{0}} M_{0}\left(1-3 \theta^{2}+2 \theta^{3}\right)
$$

quant à la détermination de la constante $k$, c'est un problème fort délicat; là encore, on peut l'ajuster expérimentalement; on peut aussi tenter de la calculer par une analyse poussée de la situation (Gyftopoulos et Steiner (1967)), mais on se trouve alors en présence de plusieurs autres constantes inconnues... 
L'interprétation précédente, qui fait intervenir deux causes fondamentales : interaction des atomes adsorbés, formant des zones où le potentiel de sortie est $\Phi_{\mathrm{A}}$, et interaction des atomes adsorbés et de la surface formant des dipôles agissant sur le potentiel de sortie, donne une expression du type :

$$
\begin{aligned}
\Phi(\theta)=\Phi_{0}-\left(\Phi_{0}-\Phi_{\mathrm{A}}\right)\left(3 \theta^{2}-2 \theta^{3}\right) \\
-k \frac{N \theta}{\varepsilon_{0}} M_{0}\left(1-3 \theta^{2}+2 \theta^{3}\right)
\end{aligned}
$$

qui rend bien compte des résultats expérimentaux, après un ajustement convenable des paramètres (fig. 10). On constate, en particulier, l'existence d'un minimum de $\Phi$, lorsque $\theta$ approche de l'unité.

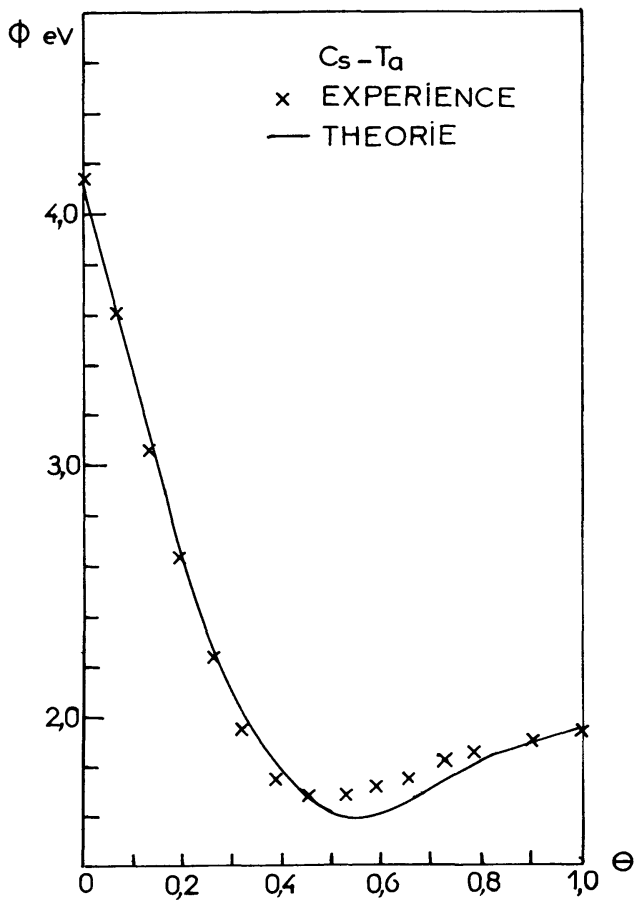

Fig. 10. - Variation de $\varphi(\theta)$ (Gyftopoulos).

La théorie précédente, due à Gyftopoulos, suppose que tous les adatomes donnent naissance à un moment dipolaire, par suite du caractère partiellement covalent de la liaison. Rasor, au contraire, suppose que seuls les ions donnent naissance à un dipôle, dont le moment est égal au produit de la charge $e$ par la distance $2 r_{\mathrm{i}}$, double du rayon (fig. 9). Le nombre $n_{\mathrm{i}}$ de ces ions par unité de surface se calcule par les méthodes du $\S 2$; on obtient alors le $\Delta \Phi$ dû à cette double couche :

$$
\Delta \Phi=-\frac{N \theta}{2 \varepsilon_{0}} 2 r_{\mathrm{i}} e \frac{1}{1+g \mathrm{e}^{-E / k T}} .
$$

Gette expression n'est exploitable que si l'on connaît la relation entre $\theta$ et $T$. L'accord avec l'expérience

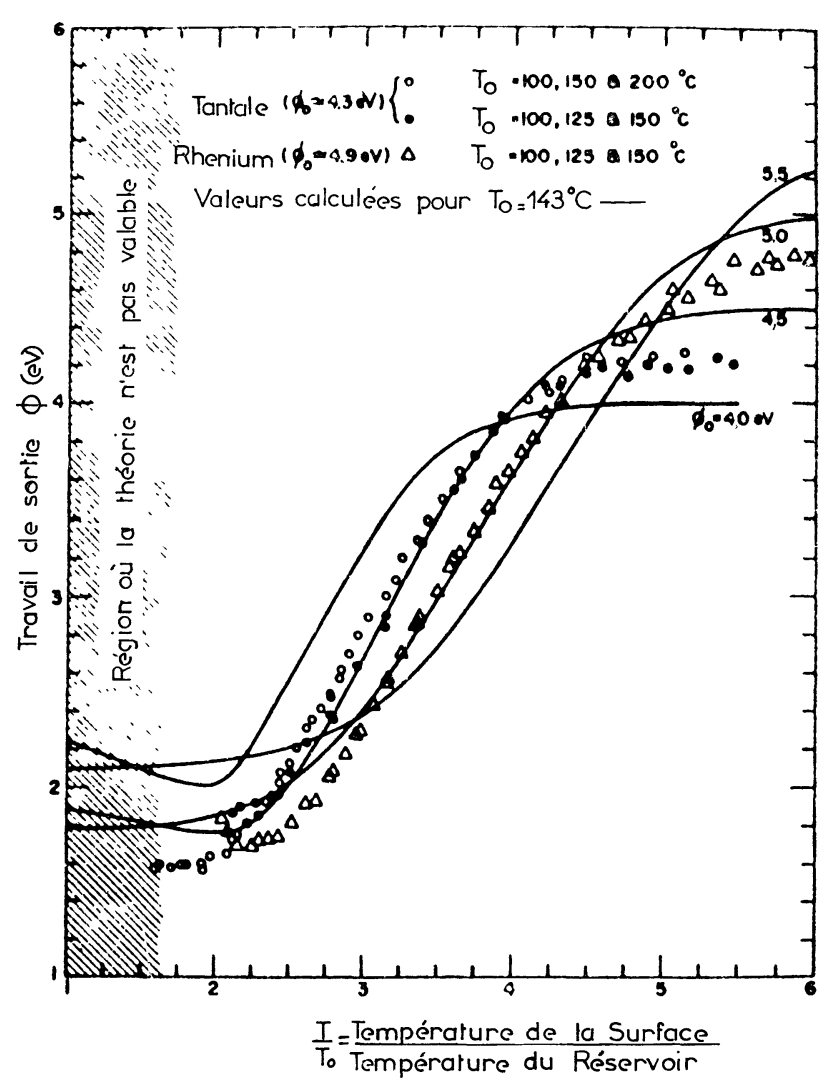

FIG. 11. - Variation de $\varphi\left(T_{0}\right)$ (Rasor).

est assez satisfaisant (fig. 11) et, en pratique, il est difficile de trouver des arguments convaincants en faveur de l'une ou de l'autre des théories.

Terminons en signalant que, dans l'une comme dans l'autre des théories qui conduisent à $\Delta \Phi$, il est possible de raffiner en tenant compte de la dépolarisation. Il est clair, en effet, que deux dipôles voisins tendent à diminuer leur moment (fig. 9). D'autre part, si la barrière $\Delta \Phi$ facilite la sortie des électrons, elle s'oppose au départ des ions; elle tend donc à augmenter $E_{\mathrm{i}}$ et, par l'intermédiaire du cycle de Rasor, agit sur E... Tous ces raffinements théoriques aboutissent à des formules très complexes (Gyftopoulos et Steiner (1967), Rasor et Warner (1964)) dont il est difficile d'apprécier le degré de validité à cause des incertitudes expérimentales sur les paramètres.

4. Les études expérimentales. - Depuis 1960, de nombreux travaux expérimentaux ont fait l'objet de publication. Le plus grand nombre de ces travaux concerne l'adsorption du césium sur le tungstène; l'importance du césium vient de son très faible potentiel d'ionisation qui rend son emploi général dans la conversion d'énergie thermo-ionique.

4.1. Les MÉthodes DE MESURE. - Le paramètre essentiel pour relier la théorie et l'expérience est la variation du potentiel de sortie $\Delta \Phi=\Phi-\Phi_{0}$ d'un 
corps dont la surface est partiellement recouverte, en fonction de la température de cette surface et la pression de la vapeur. $\Delta \Phi$ est relié au courant d'émission électronique par l'équation de Richardson et au courant d'émission ionique par l'équation de SahaLangmuir; la mesure de ces courants permet très généralement de connaître $\Delta \Phi$. D'autres propriétés habituellement utilisées pour mesurer le potentiel de sortie d'une surface propre sont également applicables au cas d'une surface partiellement recouverte d'une couche d'atomes adsorbés; la plus importante est l'émission électronique produite par effet de champ. Nordheim (1928) a montré que le courant est lié au travail de sortie par une relation assez complexe dont les éléments essentiels ont été exploités théoriquement par Good et Muller (1956). Des tentatives expérimentales ont été faites par Ehrlich et Hodda, et Hayward et Gomer (1959), mais dans le cas de surfaces recouvertes partiellement de corps électronégatifs. Utsugi et Gomer (1962) ont utilisé cette technique pour étudier et mesurer les énergies d'adsorption du baryum et du césium sur le tungstène.

Une autre propriété importante est l'émission photoélectrique dont la fréquence de seuil est reliée au potentiel de sortie. Bréaux et Médicus (1966) ont montré qu'il existait également une émission photoélectrique ionique qui pourrait être utilisée, mais cette technique nécessite encore un approfondissement théorique et expérimental.

Récemment, l'exploration d'une surface par un faisceau d'électrons a été réalisée par Haas et Thomas (1963, 1966). Le principe en est le suivant : un faisceau d'électrons très fins balaye la surface à analyser qui sert de collecteur. Le courant capté par celui-ci est une fonction du potentiel de sortie; il commande l'intensité du faisceau d'un tube de télévision dont le balayage est synchronisé avec celui du faisceau d'analyse. L'image agrandie de la surface apparaît ainsi sur l'écran.

Ges méthodes sont néanmoins rarement utilisées pour la mesure du potentiel de sortie d'une surface partiellement recouverte, et en pratique, nous nous bornerons aux résultats obtenus par les mesures de courant d'émission.

L'émission électronique ou ionique est déjà utilisée par Langmuir (1933) avec le tube à filament de la figure 1. De nombreux tubes semblables ont été utilisés avec un seul filament dans le but d'obtenir les courbes d'émission électronique en fonction de la température de la surface et de la pression de vapeur. Ges tubes nécessitent l'application d'un champ électrique entre le filament et l'anode; ils sont donc limités aux faibles pressions de vapeur pour éviter l'amorçage d'un arc. Certains tubes modernes ( fig. 2) (Lieb (1966)) sont constitués par une structure en céramique et métal et peuvent être dégazés à des températures élevées pour obtenir un vide très poussé $\left(10^{-10}\right.$ torr $)$ avant l'introduction du césium. La présence d'impuretés, même à des pressions de l'ordre de $10^{-6}$ torr
(Wilson (1967)), peut en effet modifier considérablement les propriétés de la surface. Chapman et Caufield (1964 et 1966) ainsi que Albrecht et Muz (1965) utilisent une diode très semblable à celle de Langmuir dans laquelle des anneaux de garde qui ne laissent entre eux qu'une fente fine séparent le filament $\mathrm{du}$ collecteur. Cette disposition a pour but d'éviter l'émission secondaire du collecteur dont le potentiel de sortie est très faible dans ces expériences, car il est recouvert de césium.

Le tube à deux filaments (fig. 1) sert à mesurer $\theta$. Le potentiel du filament $\mathrm{A}$, par rapport au collecteur $\mathrm{G}$ $(4 \mathrm{~V})$, est tel qu'aucun courant ne circule quelle que soit la température de ce filament. Le filament B est, au contraire, porté à un potentiel positif $(+45 \mathrm{~V})$ par rapport au collecteur; tous les ions produits sur $\mathrm{B}$ sont captés par $\mathrm{C}$. B est maintenu à une température constante de $1200 \mathrm{~K}$ et un dispositif externe compense le courant continu qui circule entre $\mathrm{B}$ et $\mathrm{C}$. $\mathrm{A}$, à partir d'une température d'équilibre basse, est brusquement porté à $1800^{\circ} \mathrm{K}$ : les atomes adsorbés s'évaporent radialement. Une partie est interceptée par B sur lequel ils s'ionisent, puis se désorbent et sont entraînés par le champ électrique vers $\mathrm{C}$. La mesure de l'impulsion de courant résultante, associée à la connaissance du diamètre des deux filaments et à leur distance, permet le calcul de $\theta$.

La diode plane fonctionne suivant le même principe que la diode cylindrique de Langmuir. Elle utilise des procédés de fabrication en usage dans la construction des convertisseurs thermo-ioniques (céramique, métal). Ces techniques permettent une très grande précision sur les dimensions géométriques des électrodes et leur espacement. Des anneaux de garde limitent exactement la surface utile. Ce type de diode permet l'étude de l'émission de surfaces constituées de poudres comprimées, d'éléments poreux ou de tubes capillaires. Dans ces deux derniers cas, le césium traverse le matériau émissif et s'ionise au cours de cette traversée.

Les émetteurs composites et les éléments poreux étudiés par Husman (1963, 1965), Gho et Shelton (1964) et Lachance et al. (1965) sont constitués de poudre comprimée d'un métal réfractaire (Mo, Ta, W) ou d'un mélange de ces métaux (Thomson et al. (1967)). Le diamètre des particules constituant la poudre, leur état de surface influe considérablement sur le diamètre moyen des pores ( 1,5 à 3,5 microns) et leur distance moyenne (4 à 17 microns). L'émetteur capillaire peut n'être constitué que d'un tube capillaire (Huber et Le Bihan (1965)) ou d'un grand nombre de tubes juxtaposés. Un émetteur de ce type, décrit par Dresser et al. (1964), résulte de l'empilement successif d'une feuille plane et d'une feuille ondulée de tungstène ou de tantale. Le diamètre des capillaires est de quelques dixièmes de millimètre et la transparence d'un tel émetteur est très supérieure à celle d'un émetteur poreux.

Un montage très riche de possibilités expérimentales consiste à percer le collecteur de la diode d'un 


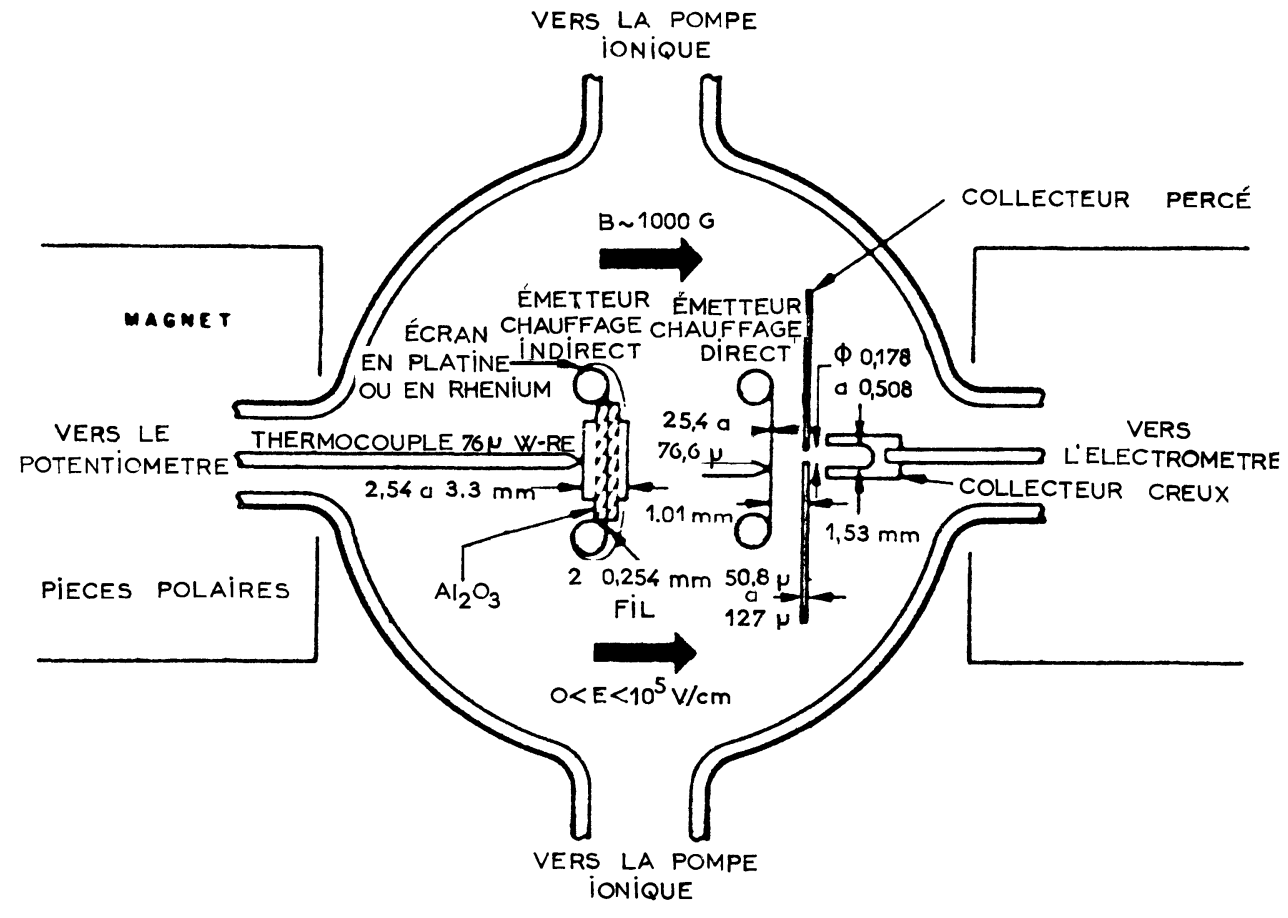

FIG. 12. - Appareil de Wilson (1966).

trou très fin derrière lequel se trouve une cage de Faraday pour éviter les émissions secondaires. Webster et Read (1964) utilisent ce principe dans un système qui constitue en même temps un microscope à émission thermo-ionique et Wilson (1966) a systématiquement étudié, par cette méthode, un grand nombre d'éléments entrant dans la fabrication de propulseur à plasma. La figure 12 représente l'appareil de Wilson. Le diamètre du trou percé dans le collecteur est de l'ordre de $0,25 \mathrm{~mm}$. Le champ électrique appliqué entre l'émetteur et le collecteur varie entre 0 et $10^{5} \mathrm{~V} / \mathrm{cm}$. Un champ magnétique de 1000 gauss canalise vers le trou les particules émises par la partie de l'émetteur située en face. L'émetteur est un ruban chauffé directement par effet Joule ou par bombardement électronique, si l'élément étudié est un conducteur; si c'est un isolant (alumine), on utilise un chauffage indirect.

Jamba et Husman (1967), Defranoult et Desplat (1968) ont réalisé un microscope électronique et ionique muni d'une optique analogue à celle des microscopes électroniques classiques. On peut ainsi observer chaque point de la surface et déduire ses propriétés du courant émis. Plusieurs auteurs ont utilisé des microscopes simplifiés tel celui de la figure 13 de Webster et Read (1964). L'émetteur est une boule de $1 \mathrm{~mm}$ de rayon environ situé à $1 \mathrm{~cm}$ d'un collecteur plan phosphorescent. Le chauffage de l'émetteur s'effectue par bombardement électronique. Comme nous l'avons indiqué au paragraphe précédent, le collecteur est

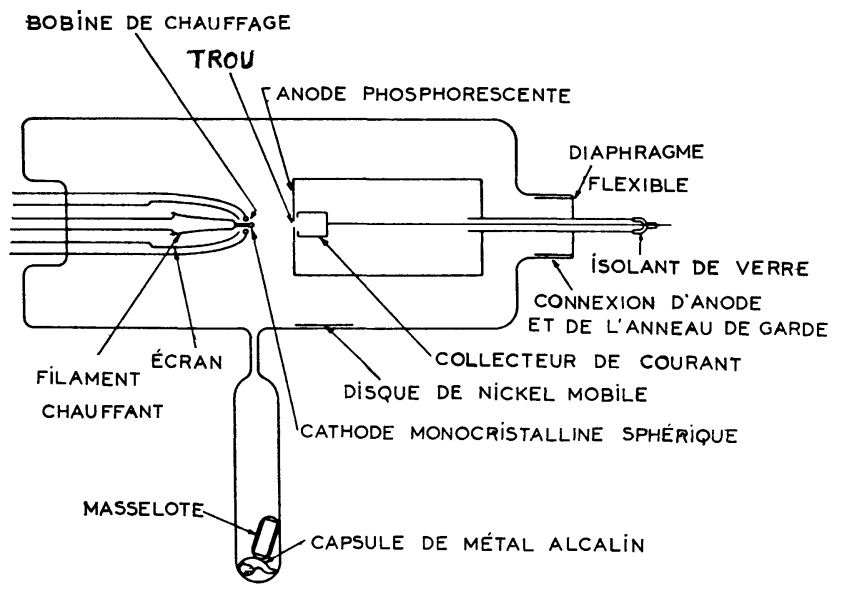

Fig. 13. - Tube de Webster et Read (1964).

percé d'un trou derrière lequel se trouve un collecteur de Faraday, l'ensemble étant mobile pour permettre l'exploration de toute l'image.

La sonde émissive a d'abord été proposée par Marchuk (1957), puis développée essentiellement par Houston (1961). La figure 14 montre un schéma du tube. Une décharge est produite dans la vapeur alcaline étudiée. Des sondes, constituées par une boucle métallique, sont plongées dans la décharge. La principale difficulté consiste à définir avec précision la surface utile de la sonde en isolant soigneusement les conduc- 


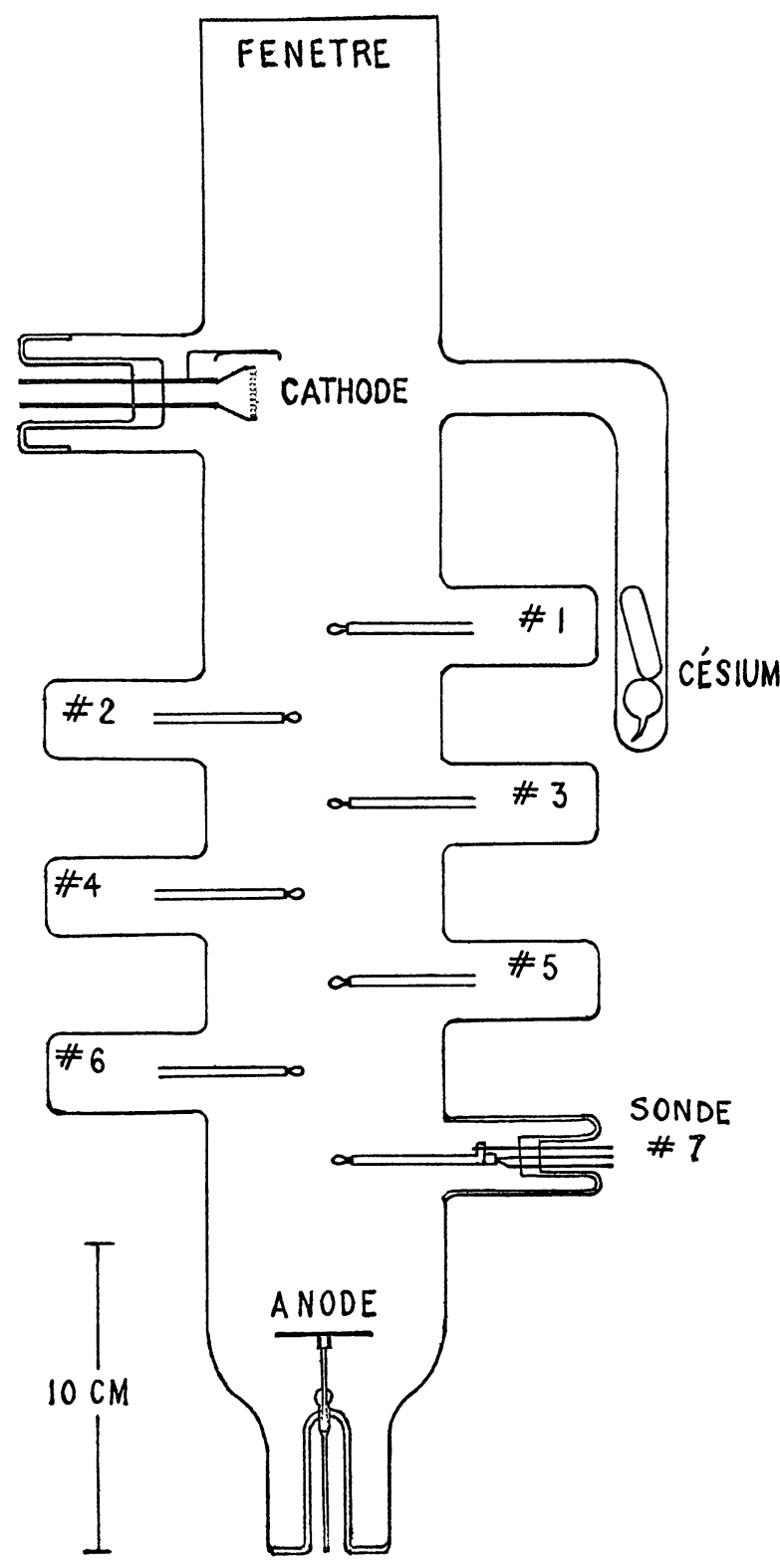

FIG. 14. - Tube de Houston (1965).

teurs. Le courant d'ions venant du plasma est d'abord mesuré par la sonde froide, porté à un potentiel négatif $(-5 \mathrm{~V})$ par rapport au potentiel flottant pour éliminer les électrons venant du plasma. La sonde se comporte alors comme une sonde de Langmuir classique. La sonde est ensuite chauffée; la variation du courant mesuré correspond au courant de saturation électronique émis par la sonde. Cette méthode permet de travailler jusqu'à des pressions de vapeur élevées car on utilise non pas le courant entre deux électrodes (entre lesquelles un arc pourrait jaillir), mais entre la sonde et le plasma qui joue le rôle de seconde électrode. De plus, des mesures comparatives sont possibles, en utilisant plusieurs sondes de natures diffé- rentes dans une même décharge. Elle a été utilisée par de nombreux auteurs, en raison de la simplicité de sa mise en œuvre et de l'étendue des mesures qu'elle permet.

4.2. Les RÉSultats expéRImentaux. - La bibliographie donne la liste des articles où figurent des mesures expérimentales récentes. Le classement est fait par type d'éléments ionisables : métaux alcalins, alcalino-terreux ou autres éléments. La plus grande partie de ces études se rapporte aux métaux alcalins et, en particulier, le césium. Nous indiquons ci-après quelques résultats particulièrement caractéristiques : leur allure générale se déduit des théories précédentes :

- En ce qui concerne l'émission d'électrons, les courbes $\log i=f(1 / T)$ seraient pratiquement des droites de pente négative, si $\varphi$ ne variait pas. La variation de ce paramètre avec la température (cf. $\S 3$ ) conduit à une courbe dite « en $\mathrm{S}$ » asymptote à deux droites, celles correspondant à $\varphi_{\mathrm{A}}$ aux basses températures et à $\varphi_{0}$ aux températures élevées;

- En ce qui concerne l'émission des ions, les courbes $\log i^{+}=f(1 / T)$, pour $\mu_{0}$ constant, présentent un palier horizontal à haute température que l'on atteint par une montée rapide lorsque la désorption commence.

La figure 15 extraite de l'article de Moizhes et Pikus (1960) donne l'allure de ces deux réseaux de

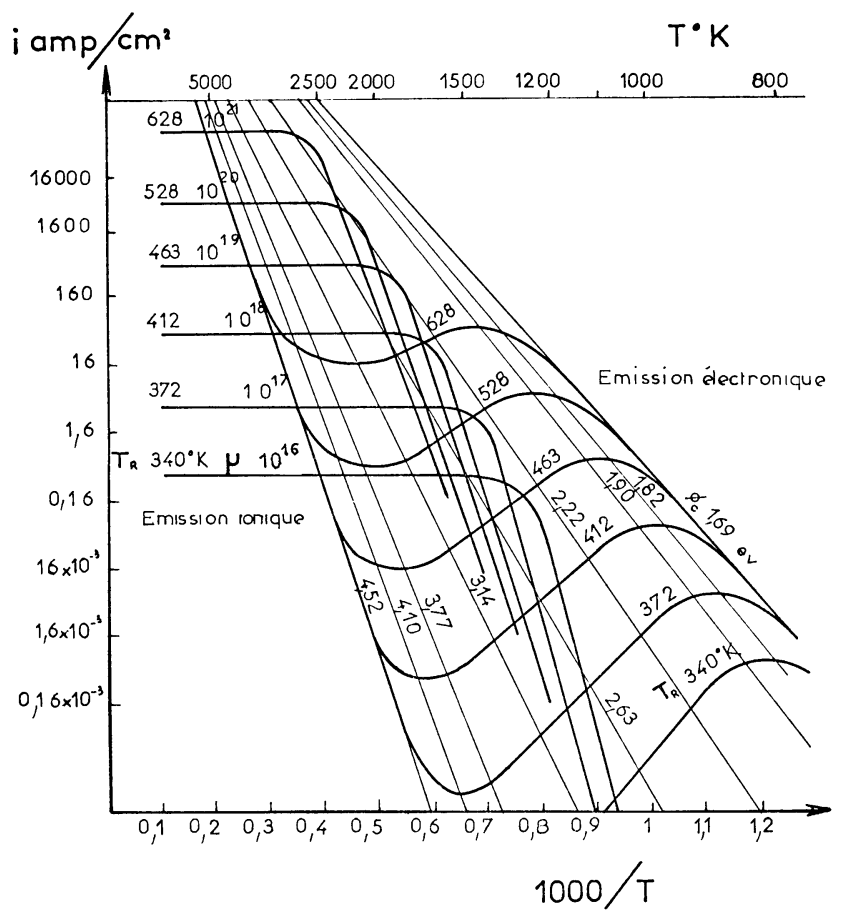

FIG. 15. - Émission ionique multipliée par $M / m$ et émission électronique d'un filament de tungstène plongé dans la vapeur de césium. 
courbes pour différentes valeurs de $\mu_{0}$. Elles sont conformes à l'allure prévue par la théorie.

Les résultats expérimentaux les plus complets ont été réalisés par Houston depuis 1961 et par Wilson (1966). Celui-ci donne un grand nombre de réseaux de courbes en $\mathbf{S}$ pour les principaux métaux, des carbures ou des métaux oxydés ainsi qu'une vingtaine de réseaux de courbes d'émission ionique de ces mêmes métaux. Ces réseaux correspondent à des flux de césium compris entre $10^{13}$ et $10^{17}$ atomes $/ \mathrm{cm}^{2} / \mathrm{s}$, ce qui correspond à des pressions de $10^{-6}$ à $10^{-3}$ torr. La figure 16 représente les courbes d'émissions élec-

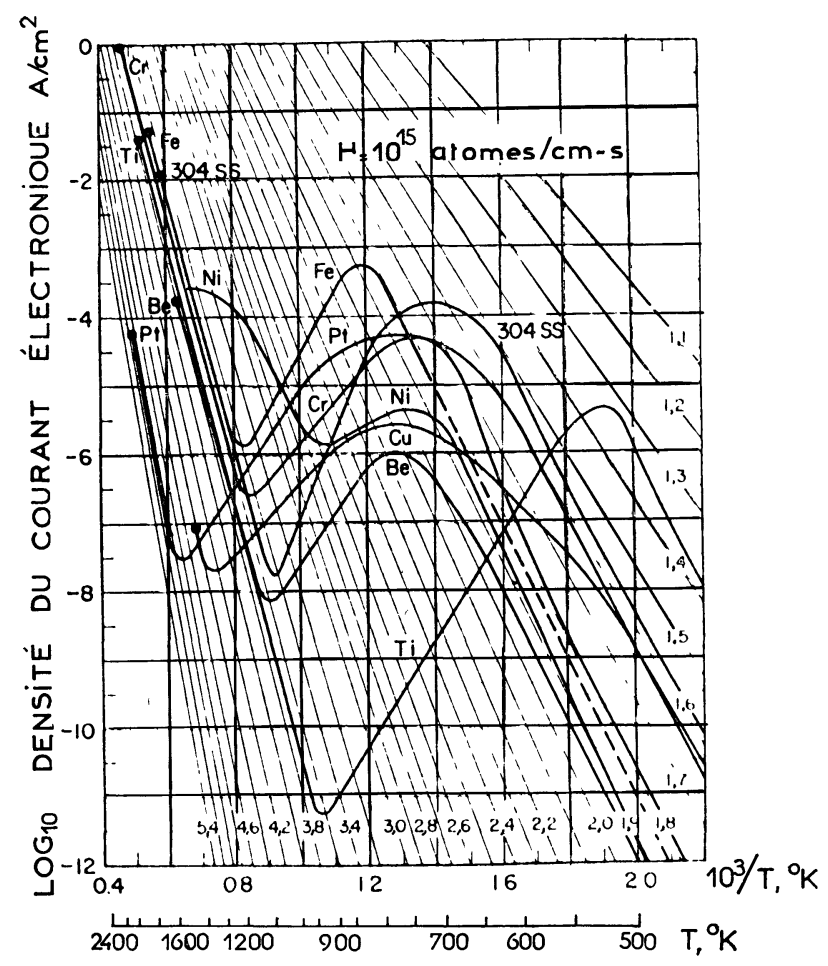

FIG. 16. - Résultat de Wilson (Cs) (1966).

troniques (Wilson (1966)) pour différents métaux à une pression de césium de l'ordre de $5 \times 10^{-6}$ torr. Cette pression correspond à une température du réservoir de césium de l'ordre de $300^{\circ} \mathrm{K}$. Wilson donne également les courbes d'émissions ioniques (fig. 17). Ces courbes présentent une hystérésis au voisinage de la saturation que Maugis (1967) interprète par la présence d'états métastables analogues à ceux observés lors de la vaporisation d'un liquide ou de la condensation d'un gaz. Lorsque la température atteint une certaine valeur, tous les atomes à l'état métastable se désorbent sous forme d'ions.

Garvin et Wilson (1965) ont étudié également l'émission de l'alumine en présence de césium : l'alumine entre, en effet, dans la fabrication des tubes ainsi que dans celle des propulseurs. Bien que l'alumine soit un isolant, on constate une émission électro-

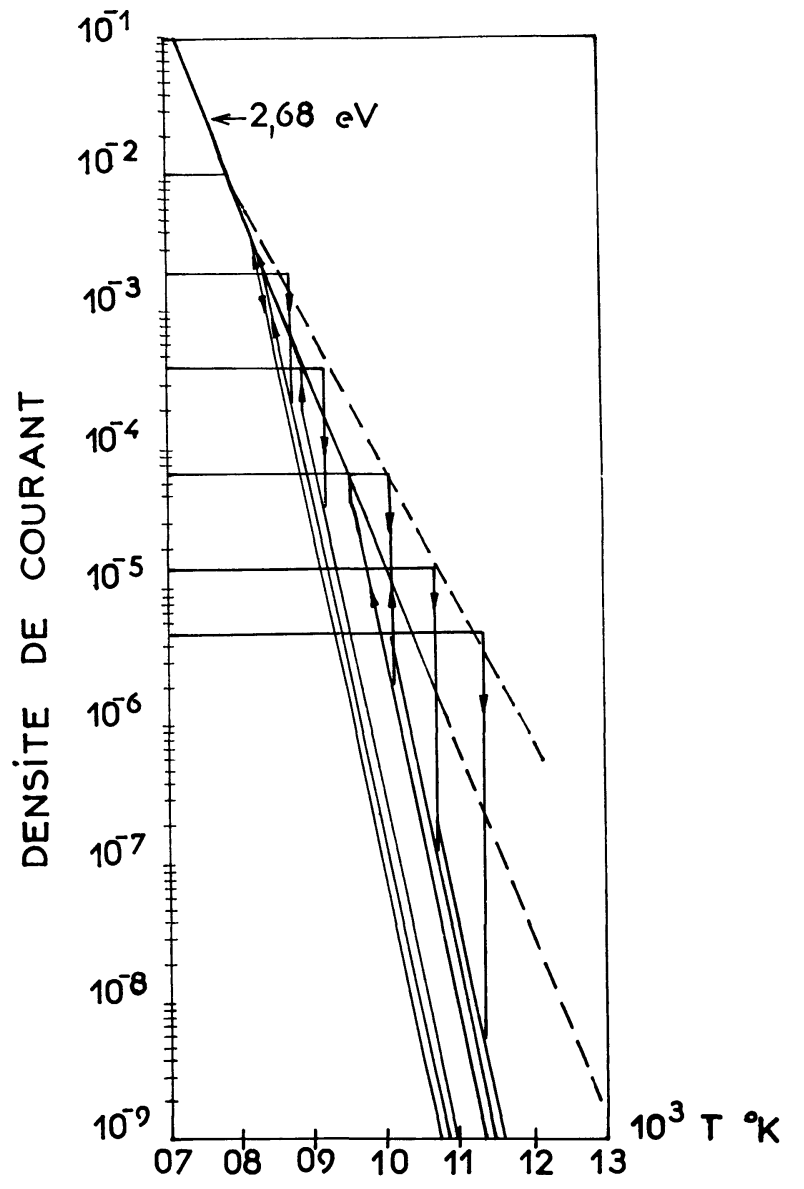

FIG. 17. - Émission ionique du césium sur le tungstène (Wilson, 1966).

nique et une émission ionique. Le sommet des courbes en $\mathrm{S}$ est, pour une même pression de césium, inférieur de deux ordres de grandeur à celui du tungstène. Les courbes d'émission ionique sont très différentes de celles observées sur les métaux et, en particulier, ne présentent pas de palier de saturation. Ceci conduit à penser que l'ionisation sur un isolant suit un processus différent de l'ionisation sur un métal. Ceci n'est pas surprenant étant donné les hypothèses sur la conductivité du support contenu dans la première partie de cet article.

Plusieurs résultats expérimentaux que nous indiquons ensuite concernent un mélange de métal alcalin et d'un autre élément : halogène ou oxygène essentiellement. L'halogène le plus employé est le fluor. La présence d'oxygène ou de fluor augmente le travail de sortie du métal émetteur, ce qui donne la possibilité d'ioniser par effet de surface des corps dont le potentiel d'ionisation est supérieur au potentiel de sortie de la surface et, dans le cas des convertisseurs thermoioniques, permet un rendement supérieur.

En réalité, le comportement d'une surface en pré- 
sence de plusieurs corps est complexe. Langpape et Minor (1964) montrent que le potentiel de sortie d'une surface en présence de fluorure de césium n'augmente qu'à partir d'une température seuil du fluorure de césium de l'ordre de 500 oK. Ce résultat est confirmé par Rasor et Gammel (1966); à cette température, la pression de vapeur du CsF est très faible ( 10 $0^{-8}$ torr $)$. Wilson (1967) a montré que l'oxygène, à des pressions aussi faibles que $10^{-6}$ torr, modifiait les courbes d'émission électronique, notamment au voisinage du maximum des courbes en $\mathrm{S}$. Ceci montre l'importance de la propreté du vide et des surfaces pour ces mesures. Houston et Dederick (1964) avaient déjà montré l'importance du dégazage de la sonde émissive avant les expériences d'émission à basse température. Ceci se conçoit facilement quand on sait qu'une monocouche d'impureté se dépose en $2 \mathrm{~s}$ à une pression de $10^{-6}$ torr.

Nous avons enfin indiqué les expériences effectuées sur les autres métaux, et, en particulier, sur le baryum. Le baryum est le métal alcalino-terreux dont le potentiel d'ionisation est le plus faible $(5,19 \mathrm{eV})$ et

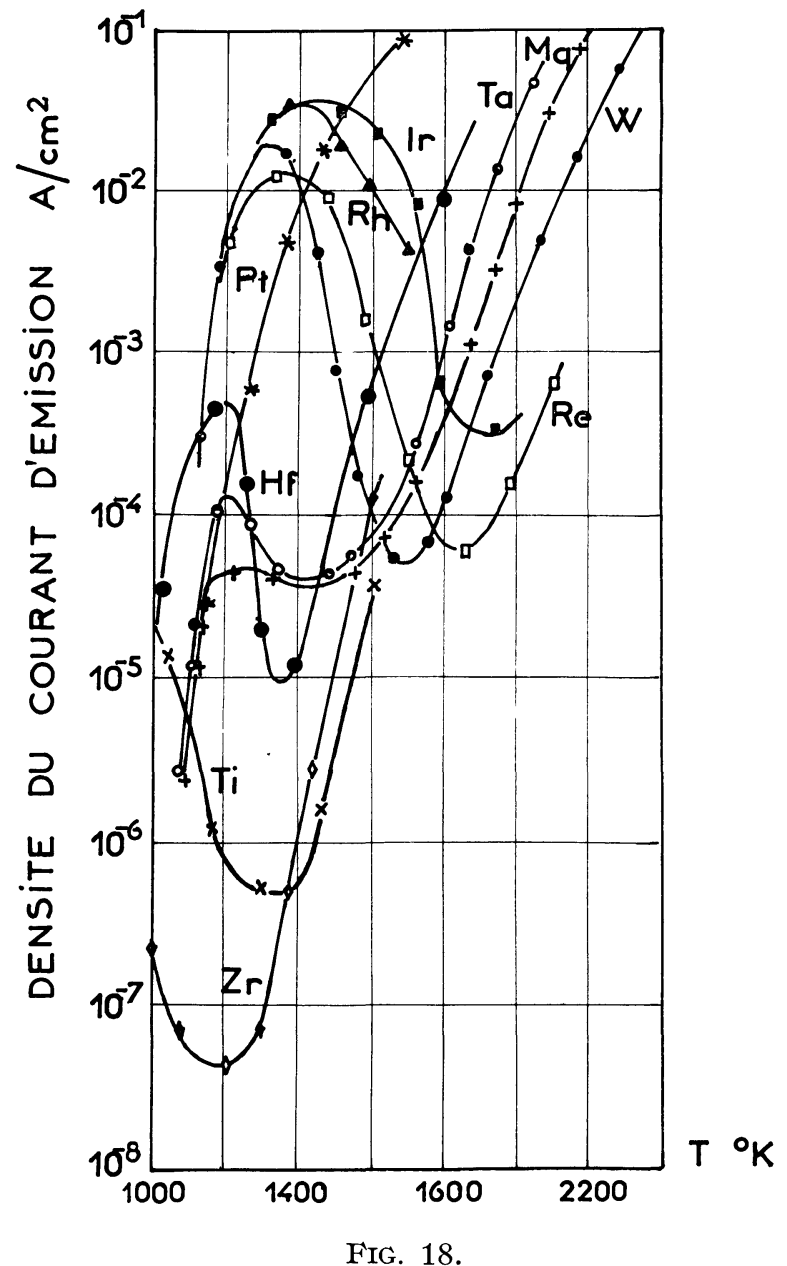

Émission en présence de baryum (Dyubua, 1965). son usage est très courant dans les divers types de cathode à oxyde. Des essais ont été réalisés pour l'utiliser dans les convertisseurs thermo-ioniques associés au césium. Une difficulté consiste à pouvoir régler indépendamment la pression de vapeur du baryum et du césium. Alleau et al. (1965) et Devin et al. (1967) suggèrent d'utiliser des composés d'insertion du graphite pour régler la pression de vapeur de césium alors que la pression du baryum (ou du lithium) est la pression de vapeur à l'équilibre avec un réservoir de métal liquide. Psarouthakis (1964 et 1965) a montré que, pour une même pression de vapeur de césium, on obtient un potentiel de sortie plus faible en présence de baryum, donc une émission électronique plus importante. Albrecht et Muz (1965) ont obtenu une surface dont le potentiel de sortie est égal à $1,4 \mathrm{eV}$ par dépôt de baryum sur une couche mince d'alumine. Utsugi et Gomer (1962) ont mesuré la variation du travail de sortie du tungstène en présence de baryum. Celui-ci passe par un minimum voisin de $2 \mathrm{eV}$ quand $\theta=1$. Ils ont également calculé la chaleur de désorption d'un atome ou d'un ion simplement ou doublement ionisé. Celle-ci varie considérablement avec le taux de recouvrement : de $-0,9$ à $+2,5 \mathrm{eV}$ pour l'atome, de 0,6 à $3,5 \mathrm{eV}$ pour l'ion $\mathrm{Ba}^{++}$et de 1,9 à $3,5 \mathrm{eV}$ pour l'ion $\mathrm{Ba}^{+}$lorsque $\theta$ diminue de 0,45 à 0 . Dyubua (1965) donne les courbes d'émission électronique d'un grand nombre de métaux en présence de baryum ( fig. 18). Cette figure montre que, suivant la température, certains corps doivent être utilisés préférentiellement suivant que l'on désire obtenir ou, au contraire, exclure une émission électronique : aux basses températures (entre 1400 et 1800 oK), l'émission du rhénium est supérieure à celle du tungstène; au contraire, le zirconium au-dessous de $1400^{\circ} \mathrm{K}$ a une émission très faible.

Conclusion. - Il est possible d'obtenir des sources d'ions par émission thermique en mettant en œuvre l'ionisation de contact. Les densités de courant que l'on peut obtenir sont du même ordre de grandeur que les densités de courant électronique données par effet Richardson. On peut donc envisager (Maugis (1967)) d'utiliser ces sources, soit comme source d'électrons, soit comme source d'ions en les plaçant en face d'un collecteur convenablement polarisé. On peut également les utiliser comme émetteur mixte fournissant les éléments nécessaires à la synthèse d'un plasma. C'est sur cette méthode que fonctionne un grand nombre de machines à plasma signalées dans l'introduction. Cependant, dans ce cas, il convient de tenir compte de la zone de transition ou gaine qui se forme entre le plasma et la surface émissive. Dans cette zone, se trouvent les particules venant de la surface, mais également celles venant du plasma. L'équation de Saha appliquée à la limite plasmagaine, celles de Saha-Langmuir appliquées à la surface conduisent à la détermination de la charge d'espace dans la gaine. Celle-ci est positive ou négative suivant 
les conditions de fonctionnement, mais, de toute façon, crée un champ électrique à la surface et modifie l'émission de celle-ci par effet Schottky. Ce champ régule le flux de particule qui atteint le plasma de façon à obtenir finalement la neutralité. L'étude devient fort délicate et nous nous bornons à renvoyer le lecteur à l'article de Morris (1968) qui traite cette question en détail.

\section{BIBLIOGRAPHIE}

Ia première partie de cette bibliographie rassemble tous les articles cités dans le texte à l'exception de ceux qui concernent directement les résultats expérimentaux. Ceux-ci sont regroupés après la bibliographie générale sous différentes rubriques (métaux alcalins purs, métaux alcalins associés à d'autres éléments et, en particulier, des halogénures, métaux alcalino-terreux). Nous avons utilisé les abréviations traditionnelles et également les abréviations suivantes :

T.C.S.C. : Thermionic Conversion Specialist Conference. T.E.P.G. : Thermionic Electrical Power Generation.

Alleau (T.), Devin (B.), Durand (J. P.) et Lesueur (R.), T.E.P.G., sec. 3 b, 1965.

Bennetit (A. J.) et FAlicon (L. M.), Phys. Rev., 1966, $151,2,512$.

Boutry (G. A.), Physique appliquée aux industries du vide et de l'électronique, Masson, 1962.

Bowers (H. G.) et Wolga (G. J.), J. Appl. Physics, 1966, 37, 5, 2024.

Bréaux (O. P.) et Médicus (G.), Tech. Rep. AFAL TR, 1966, 66, 113.

Carabateas (E. N.), J. Appl. Physics, 1962, 33, 9, 2698.

Chо (A. Y.) et ShelTon (H. S.), A.I.A.A. Aerospace Sciences Meeting, N. Y., preprint no 64-11, 1964.

Defranoulit (P.) et Desplat (J. L.), J. Physique (à paraître).

DEVIN (B.), LESUEUR (R.) et SETTON (R.), T.C.S.C., sec. II B-5, 1967.

DoucerT (H.), Thèse, Paris, 1966.

Doucet (H.) et Lamain (H.), Rev. Phys. Appl., 1967, 2, $3,153$.

Dresser (D. L.), Laurita (W. G.), Dunlop (J. D.), Huber (H.) et LE BIhAN (R.), A.I.A.A.J., 1964, 2, 1,52 .

Eberhagen (A.), Fortsch. Physik, 1960, 8, 245-294.

EHRLICH (G.) et HODdA (F. G.), J. Chemical Physics, 1959, 30, 2.

EnRiQues (L.) et MAGistrelili (F.), Rev. Scient. Instrum., 1964, 35, 12, 1708.

Felden (M.), Patou (L.) et Haug (R.), Rev. Phys. Appl., 1966, 1, 48.

Gadzuk (J. W.) et Carabateas (E. N.), J. Appl. Physics, 1965, 36, 2, 357.

GILLEO (M. A.) et KASH (S. W.), J. Am. Roc. Soc., 1961, 31, 621 .

Von Goeler (S.), OHE (T.) et D'ANGelo (N.), Plasma Physics Lab., Princeton, Matt., 1965, 357.

Von Goeler (S.), Ohe (T.) et D'Angelo (N.), J. Appl. Physics, 1966, 37, 6, 2519.

GOMER (R.), Discussions of the Faraday Soc., 1966, 41, 14.

Good (R. H.) et MülLER (E. W.), Hand. Physik, 1956, 21, 190
Grimley (T. B.), J. Phys. Chem. Solids, 14, 227, 1960. GuIlıNo (E.), Inst. für Plasma Physik, Garching, Rapp. IPP 2/20, 1963.

Gyftopoulos (E. P.) et LEvine (J. D.), J. Appl. Physics, 1962, 33, 1, 67.

GyFtopoulos (E. P.) et STEINER (E.), Report on the 27th Ann. Conf. Phys. Elec., Cambridge, 1967.

Hass (G. A.) et Jensen (J. T.), J. Appl. Physics, 1960, 31, 7.

Hass (G. A.) et Thomas (R. E.), J. Appl. Physics, 1963, 34, 3457.

HaAs (G. A.) et Thomas (R. E.), Surface Science, 1966, $4,1,64$.

Hayward (D. O.) et Gomer (R.), J. Chem. Physics, 1959, 30, 1617.

HERNQVist (K. G.) et LEvine (J. D.), R.C.A. Rev., 1966, 27, 1, 140.

Honig (R. E.), R.C.A.Rev., 1962, 567.

Houston (J. M.), Advanc. Electron., 1962, 17, 125.

Huber (H.) et LE Bthan (R.), Combustion and Propulsion Panel, Agard, Nato, Athènes, 1963.

Husman (O. K.), Jamba (D. M.) et Denisson (D. R.), A.I.A.A. J., 1966, 4, 2, 273.

Husman (O. K.) et Turk (R.), A.I.A.A. J., 1965, 3, 1553.

Jamba (D. M.) et Husman (O. K.), J. Appl. Phys., 1967, 38, 6, 2630.

KAMINSKI (M.), Atomic and ionic impact phenomena on metal surface, Springer-Verlag, Berlin, 1965.

KAPLit (M.), Schrenk (G. L.) et ZELBY (L. W.), Advanc. Energy Conversion, 1967, 7, 177.

KoENig (D. R.) et Pigford (T. H.), Rapp. U.C.R.L. 16804, Université de Californie, Berkeley (Cal.), 1966.

Lachance (M.), KUSKevics (G.) et Thompson (B.), A.I.A.A. J., 1965, 3, 8, 1498.

Langmuir (I.), J. Am. Chem. Soc., 1932, 54, 1252.

LANGMUIR (I.), J. Am. Chem. Soc., 1932, 54, 2798.

LAANGMuIR (I.), Phys. Rev., 1933, 43, 224.

LANGmuir (I.) et Kingdon (K. H.), Proc. Roy. Soc. (Lond.), 1925, A 107, 61.

LEVINE (J. D.) et PH. (D.), Thèse M.I.T., 1963.

LEVINE (J. D.) et GYFTOPOULOS (E. P.), Surface Science, $1964,1,171,225,349$.

LiEB (D. P.) et Miskolczy (G.), T.C.S.C., 1964, 22.

Marchuk (M. P.), Radio Eng. Electr. Phys., 1957, $2,13$.

MaUGis (D.), Thèse Orsay (Paris), 1967.

MÉdicus (G.) et BrÉAUX (O. P.), Rep. no 26th Annual Conf. Phys. Electron., M.I.T., 1966.

Moizhes (B. Ya) et Pikus (G. E.), Sov. Phys. Solid States, 1960, 2, 523.

Moreau (J. B.), J. Physique, 1965, 25, 447.

Moreau (J. B.), J. Physique, 1968, Colloque de Grenoble.

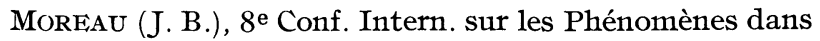
les Gaz ionisés, Vienne, 1967, 145. 
Morris (J. F.), J. Appl. Physics, 1968, 39, 3, 1705.

Mulitiken, J. Chem. Physics, 1955, 23, 1833, 1841, $2338,2343$.

NORDHEIM (L. W.), Proc. Roy. Soc. (Lond.), 1928, A 121, 626.

Pauling (L.), The Nature of the Chemical Bond, Cornell University Press, Ithaca, N. Y., 1960, p. 82.

RASOR (N. S.), P.I.E.E.E., 1963, 51, 733.

RASOR (N. S.), CARTER (R. L.), MCKISSON (R. L.), VERNON (R.) et WARNER (C.), Atomics International Report AI. 6799, 1961.

Rasor (N. S.) et Warner (C.), J. Appl. Physics, 1964, 35, 9, 2589.

Rititneir (E. S.) et Milch (A.), J. Appl. Physics, 1962, 33, 1, 228.

RYNN (N.) et D'ANGELO (N.), Rev. of Scient. Instr., 1960, 31, 12, 1326.

SChEER (M. D.) et Fine (J.), J. Chem. Physics, 1962, 37, 1, 107.

SChEER (M. D.) et Fine (J.), J. Chem. Physics, 1963, 38, 2, 307.

Slater (J. C.), Introduction to Chemical Physics, McGraw-Hill Book Co., Inc., N. Y., 1963.

SteineR (D.) et Gyftopoulos (E. P.), Report on the 27th Ann. Conf. Phys. Elec., Cambridge, 1967.

STEINER (D.) et GyFtopoulos (E. P.), T.C.S.C., sec. IC 2, 1967.

TAylor (J. B.) et Langmuir (I.), Phys. Rev., 1933, 44, 6, 423.

TAylor (J. B.) et LANGmuir (I.), Phys. Rev., 1937, 51, 753.

Thompson (B.), Kuskevics (G.) et Lachance (M.), A.I.A.A. J., 1967, 5, 1168.

WAdA (J. Y.) et KNEChTLI (R. C.), Proc. IRE, 1961 49, 1926.

Zandberg (E. J.) et Ionov (N. I.), Sov. Phys. Uspehki, 1959, 2, 255

Études générales de l'ionisation du césium ou éventuellement d'un autre métal alcalin sur plusieurs métaux réfractaires. Les méthodes utilisées sont essentiellement la sonde émissive, la diode simple ou munie d'un collecteur de Faraday.

Garvin (H. L.), Ad. En. Conv., 1962, 2, 375.

GaRvin (H. L.) et WiLson (R. G.), A.I.A.A. J., 1965, 3, $10,1867$.

GOTTLIEB (M.), ZOLLWEG (R. J.), RICHARDSON (L. S.), FEIN (A. E.) et Destresse (J. G.), Tech. Document. Report ASD TDR 62-1082, 1963.

Houston (J. M.), Thermoionic Converter Contractors Meeting, Bedford, Mass., 1961.

Houston (J. M.), Bull. Amer. Phys. Soc., série II, 1961, 6, 358.

Houston (J. M.), Gen. Elec. Res. Lab., Rep. 65 RL $3963 \mathrm{E}, 1965$.

Houston (J. M.), T.C.S.C., 1966, 333.

Houston (J. M.) et DEDERICK (P. K.), T.C.S.C., 1964, 77.

Houston (J. M.) et Dederick (P. K.), Gen. Elec. Res. Lab., Rapp. 65 RL 3872 E, 1965.
Houston (J. M.) et WeBster (H. F.), Advanc. Electron., 1962, 17, 145.

Husman (O. K.), A.I.A.A. J., 1963, 1, 2607.

Husman (O. K.), Progress in Austronautics and Aeronautics, 1963, 9, 145.

Husman (O. K.), Electrostatic Propulsion Conf., Monterey, 1960.

LIEB (D.), KITRILAKIS (S. S.) et WeINSTEIN (J. H.), Thermo-Electron. Eng. Corp., Contract NAS 3-2539, 1963-1964.

Webster (H. F.) et REAd (P. L.), Surface Science, 1964, 2, 200.

WILSON (R. G.), Final Summury, Report contract NAS 3-5278, NASA CR, 1966, 54, 680.

WILSON (R. G.), J. Appl. Physics, 1966, 37, 3161.

Études plus fragmentaires concernant l'action d'un alcalin sur un ou deux métaux. Les méthodes utilisées sont plus disparates : diode cylindrique, photoémission, potentiel de contact.

Charkovskir (E. F.) et PTITsyn (G. V.), Soviet Physics Technical Physics, 1965, 10, 410 (K sur Pt).

Desteese (J. G.), Appl. Phys. Lett., 1963, 25-26 (Cs sur $\mathrm{Re})$.

DrESSER (D. L.), T.C.S.C., 1964, 53 (Cs sur W et Re).

FENDLEY (J. R.), T.C.S.C., 1964, 71.

FIRLE (T. E.), Bull. Amer. Phys. Soc., série II, 1961, 6, 358 (Cs sur Ta et Mo).

Harter (F.), Darrah (J. G.) et Bolan (P.), T.C.S.C., 1963, 206 (Cs sur W, Re, Ta).

Jensen (C. G.), Campbeli (R. E.) et Dong (W. D.), T.C.S.C., 1963, 192 (Co sur Mo).

KenNedy (A. J.) et Trimmer (D. S.), T.C.S.C., 1964, 63 (Cs sur Ru, Ir, Re).

KIDD (P. W.), J. Appl. Physics, 1965, 36, 14 (Cs sur UC, W).

LEBEDEV (S. Ya), Stavisskil (Yu, Ya) et SHuT'Ko (Yu V.), Soviet Physics Technical Physics, 1962, 6, 836 (Cs sur Mo poreux).

LEE (T. J.), BLOTT (B. H.) et Hopkins (B. J.), T.E.P.G., sec. 6, 1965 (Cs sur W).

MyatT (J.), Advanc. En. Conv., 1963, 3, 279 (Cs sur ZrC).

RASOR (N. S.), KITRILAKIS (S. S.) et LIEB (D. P.), T.C.S.C., 1963, 169 (Cs sur W, Ta).

Stavisskil (Yu, Ya) et LeBedev (S. Ya), Sov. Phys. Tech. Phys., 1960, 5 (Cs sur W poreux).

SWANSON (L. W.), STrayer (R. W.) et CharbonNiER (F. M.), Surface Science, 1964, 2, 177 (Cs sur W, Mo).

WeBster (H. F.), J. Appl. Physics, 1961, 32, 1802 (Cs et $\mathrm{Rb}$ sur $\mathrm{Ta}$ ).

WEBSTER (H. F.), T.C.S.C., 1963, 187 (Cs sur Ta)

WEBSTER (H. F.), Gen. Elect. Rap. AF CRL 67-0334, 1967 (Cs sur $\mathrm{Re}$ ).

Webster (H. F.), J. Appl. Physics, 1967, 38, 9, 3700 (Cs sur $\mathrm{Re}$ ).

ZANDberg (E. J.), PALEW (V. I.) et TonTEgode (A. Ya), Sov. Phys. Techn. Physics, 1962, 7, 147 (Cs sur W).

Zukoski (E. E.) et PinchaK (A. C.), A.I.A.A. J., 1965, $3,2,270(\mathrm{~K}$ sur $\mathrm{W})$. 
Études plus particulières de certains phénomènes de surface en présence de métal alcalin.

Antypas (G. A.), Tinder (R. F.) et Donalddon (E. E.), J. Appl. Physics, 1968, 39, 4, 1967 (Na, K sur $\mathrm{Fe})$.

BAUER (K.) et Bloss (W.), T.E.P.G., sec. 6, 1965 (Cs sur film $\left.\mathrm{Al}_{2} \mathrm{O}_{3}\right)$.

BLoss (W.) et MuZ (E.), T.C.S.C., 1966, 382 (Cs sur $\left.\mathrm{Al}_{2} \mathrm{O}_{3}(30 \AA)\right)$.

Caufieid (H. F.), Chapman (R. A.) et Hemstreet (H. W.), T.C.S.C., 1963, 228 (Cs sur $\mathrm{Al}+\mathrm{Al}_{2} \mathrm{O}_{3}$ ).

Caufield (H. F.) et Chapman (R. A.), J. Appl. Physics, 1966, 37, 13, 4927 (Cs sur CsSb).

Chapman (R. A.), J. Appl. Physics, 1964, 35, 10, 2832 (Cs sur $\mathrm{Al}+\mathrm{Al}_{2} \mathrm{O}_{3}$ ).

MCFarLand (R. H.) et Kinney (J. D.), A.E.C. Contract $\mathrm{n}^{\circ} \mathrm{W}$-7405-eng-48, 1965, University of California ( $\mathrm{Li}$, $\mathrm{Na}$ sur $\mathrm{W}$ ).

KirkPATRICK (M. E.) et MENDELSON (R. A.), A.I.A.A. J., 1967, 5, 3, 562 (Cs sur CsSb).

Koenig (D. R.) et Pigford (T. H.), Rapp. UCRL 19804 et 16805, Rapp. conf. 660324, 1-2, University of California, Berkeley, Cal., 1966 (Cs sur W (100)).

LE Bihan (R.) et MaUgis (D.), Ann. radioélectr., 1965, 80, 126-158 (Cs sur Ta capillaire).

Levine (J. D.), J. Appl. Physics, 1966, 37, 5, 2175 (Cs sur saphir).

MAYER (H.), Rev. Soc. royale belge des Ingénieurs, 1965, 5, 231 (Cs, Li, Na sur verre).

MuZ (E.) et KLUGE (W.), T.C.S.C., sec. IC, 1967, 5. Muz (E.), Peter (E.) et Bloss (W.), T.E.P.G., sec. 3 b, 1965.

UTSUGI (H.) et GoMER (R.), T.C.S.C., sec. IC 5, 1967 (Cs sur W).

Résultats concernant les métaux alcalins associés à des halogénures alcalins.

AAMOdT (R. L.), BRown (L. J.) et Nichols (B. D.), J. Appl. Physics, 1962, 33, 6, 2080-2085 (Cs, CsF sur Mo).

Ionov (N. I.), Sov. Phys. Tech. Physics, 1956, 26, 2314 $(\mathrm{CsCl}, \mathrm{KCl}$ sur $\mathrm{W})$.

Ionov (N. I.) et KaraTaEv (V. I.), Sov. Phys. Tech. Physics, 1962, 7, 454 ( $\mathrm{KCl}$ sur W, Ta).

KAPLIT (M.) et Schrenk (G. L.), T.C.S.C., San Diego, 1965 (Cs, CsF sur Mo).

LANGPAPE (R.) et MiNOR (A.), T.C.S.C., 1964, 87 (Cs, CsF sur $\mathrm{Nb}, \mathrm{Ta}, \mathrm{Mo}, \mathrm{W}, \mathrm{Re})$.

LANGPAPE (R.) et Minor (A.), T.E.P.G., sec. 3 b, 1965 (Cs, CsF sur Nb, Ta, Mo, W, Re).

LIEB (D.) et KITRILAKIS (S. S.), T.C.S.C., 1966, 340 (CsF sur W).

RANken (W. A.), AamodT (R. L.), Brown (L. J.) et Nichols (B. D.), Ad. En. Conv., 1963, 3, 235 (Cs, $\mathrm{CsF}$ sur Mo).

Rasor (N. S.) et GammeI, (G.), T.C.S.C., 1966, 324 $(\mathrm{Cs}+\mathrm{CsF}$ sur W).
SkeEn (C. H.), J. Appl. Physics, 1965, 36, 1, 84 (Cs, CsF).

WhITE (J. E.), Surface Science, 1967, 7, 93 (NaI, CsI sur $\mathrm{W})$.

Zandberg (E. J.), Sov. Phys. Techn. Physics, 1957, 2, $2399(\mathrm{~K}, \mathrm{KCl}, \mathrm{CsCl}$, sur W).

ZANDBERG (E. J.) et TONTEGode (A. Ya), Sov. Phys. Techn. Physics, 1965, 10, 858 (Li, Na, K, Cs $+\mathrm{LiCl}$, $\mathrm{NaCl}, \mathrm{KCl}, \mathrm{CsCl}$ sur $\mathrm{Re})$.

Résultats concernant les métaux alcalins associés à d'autres éléments $\left(\mathrm{H}, \mathrm{H}_{2}, \mathrm{Ba}, \mathrm{Sr}, \mathrm{CCl}_{2}, \mathrm{Cl}_{2}, \mathrm{O}_{2}\right)$.

FEHRS (D. L.) et STICKNEy (R. E.), Surface Science, 1967, 8, $267\left(\mathrm{Cs}, \mathrm{O}_{2}, \mathrm{H}_{2}\right.$ sur Ta).

KITRILAKIS (J.), LIEB (D.), RUFEH (F.) et VAN SOMEREN (L.), Thermo. Electron. Contract, 1966, 951-962 $\left(\mathrm{CsF}, \mathrm{Cs}+\mathrm{Cu}_{2} \mathrm{O}\right.$ ou $\mathrm{Cs}_{2} \mathrm{O}$ sur $\left.\mathrm{W}\right)$.

Long (J. D.) et Psatourakis (J.), T.C.S.C., 1966, 355 (Cs, Cs $+\mathrm{Ba}$ sur $\mathrm{Re}, \mathrm{Mo}, \mathrm{Ru}, \mathrm{Nb}, \mathrm{Nb}$ oxydé).

Missman (R. A.) et GeHman (B. L.), Advanc. En. Conv., 1963, 3, 229 (Cs, Cs + H sur Mo).

Psarouthakis (J.), T.C.S.C., 1964, 100 (Cs + Ba, $\mathrm{Cs}+\mathrm{Sr}$ sur Mo, W).

PSARouthakis (J.), T.E.P.G., sec. 3 b, 1965.

Psarouthakis (J.), A.I.A.A. J., 1966, 4, 1201 (Cs + Ba).

Rekova (L. P.), STrel, Chenko (S. S.), FogeL (Ya M.) et Sun'shan (K.), Radiotechknika I Electronika, 1964, 9, $1\left(\mathrm{Na}, \mathrm{K}+\mathrm{CCl}_{4}, \mathrm{Cl}_{2}, \mathrm{O}_{2}, \mathrm{H}_{2}\right.$ sur W).

Rump (B. S.), Bryant (J. F.) et Gehman (B. L.), T.C.S.C., 1963, $236\left(\mathrm{Cs}+\mathrm{CsH}, \mathrm{Cs}+\mathrm{H}_{2}\right.$ sur Mo, $\mathrm{Ni}, \mathrm{W})$.

SASAli (N.) et ONOHI (M.), Nature, 1954, 174, $84\left(\mathrm{CCl}_{4}\right.$, $\mathrm{CHCl}_{3}$, fréon sur $\mathrm{Pt}$ ).

WILson (R. G.), Surface Science, 1967, 7, 157 (O, Cs, Cs + O sur Ta).

Résultats concernant les métaux alcalino-terreux et d'autres éléments (Co, $\mathrm{Hg}$, Th).

Al,BREChT (H.) et MUZ (E.), T.E.P.G., sec. 6, 1965 (Ba sur $\mathrm{Al}_{2} \mathrm{O}_{3}$ ).

Brown (H. M.) et Hensley (E. B.), J. Appl. Phys., 1966, 37, 7, 2874 (Ba).

Douce'T (H.) et GRÉSILlon (D.), Rap. PMI 324 (École Polytechnique), 1966 (déc.) (In sur Ir).

Dyubua (B. Ch.), Radio Engineering and Electronic Phys., 1965, 6, 999 (Ba sur Mo, W, Re, Ir, Pt, Zr, Hf, Ti).

Dyubua (B. Ch.) et Popov (B. N.), Radio-Eng. Electr. Phys., 1962, 7, 1454 (Ba sur Rh, Ir, Pt, Re, Ti, Zr, Hf, $\mathrm{W}+\mathrm{O})$.

Estrup (P. J.), ANDERson (J.) et DANForTh (W. E.), Surface Science, 1966, 4, 286-298 (Th sur W).

Gavriluk (V. M.) et Medvedev (V. K.), Soviet. Phys. Solid State, 1963, 4, 9 (Ba et Co sur W). 
GRIEGER (G.), Inst. für Plasmaphysik, Garching, Rapp. IPP 2/47, 1966 (Ba sur Re).

Haques (C. A.) et Donaldson (E. E.), Rev. Sci. Inst. 1963, 34, $409(\mathrm{Al}+\mathrm{Cu}$ sur $\mathrm{W}+\mathrm{O})$.

IoNov (N. I.) et MiTrSev (M. A.), Sov. Phys. J.E.T.P., 1960, 11, 972 (Nd et Pm sur W).

Kranz (A. Z.), Physics of Fluids, 1962, 5, 9 (Ba sur Re).

MAYER (H.), Rev. Soc. royale belge des Ingénieurs, 1965, 5, 231 (Hg sur verre).

MoRdIN (A. M.), P.M.I. 325 (École Polytechnique), 1966 (He* sur Ir).

REynolds (F. L.), Journ. Chem. Phys., 1963, 39, 4, 1107 (Sr, Ca sur W).
Rititner (E. S.) et Levi (R.), J. Appl. Phys., 1962, 33, 2336 (Ba sur W et Mo).

Rynn (N.), Hinnov (E.) et Johnson (L. C.), Rev. of Scient. Instrum., 1967, 38, 10, 1378 (Ba sur Re).

Szhenov (Yu K.), Sov. Phys., J.E.T.P., 1960, 37 (10) 2 , $239(\mathrm{Ba}, \mathrm{Sn}, \mathrm{Ca}, \mathrm{Mg}$ et $\mathrm{Na}$ sur W).

ZINGERman (Y. P.) et Ishchuk (V. A.), Sov. Phys. Solid State, 1963, 4, 8, 1618 (Ba sur W).

ZINGERMAN (Y. P.), IshchuK (V. A.) et MoRozovskiI (V. A.), Sov. Phys. Solid. State, 1960, 3, 2030 (Ba sur W).

ZINGERMAN (Y. P.), IshchuK (V. A.) et MorozovsKII (V. A.), Sov. Phys. Solid State, 1961, 3, 4, 760 $(\mathrm{Ba}, \mathrm{Mg}, \mathrm{Be}$ sur $\mathrm{W})$. 